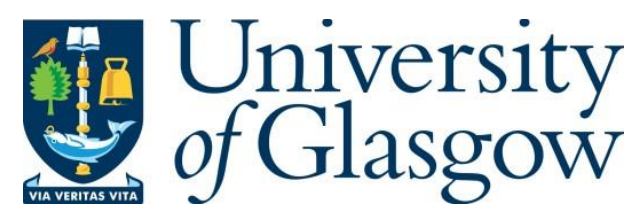

Rasethuntsa, T. R. and Ansari, I. S. (2020) Applications of Meijer's factorization theorems in performance analyses of all-optical multi-hop FSO systems. IEEE Transactions on Wireless Communications, (doi: 10.1109/TWC.2020.3038944).

There may be differences between this version and the published version. You are advised to consult the publisher's version if you wish to cite from it.

http://eprints.gla.ac.uk/226705/

Deposited on: 4 December 2020

Enlighten - Research publications by members of the University of Glasgow http://eprints.gla.ac.uk 


\title{
Applications of Meijer's Factorization Theorems in Performance Analyses of All-Optical Multi-Hop FSO Systems
}

\author{
Tau Raphael Rasethuntsa, Imran Shafique Ansari
}

\begin{abstract}
The use of bivariate Fox H-functions (BFHFs) in performance analyses of wireless communication systems has gained considerable attention in past few decades. However, the non-existence of robust built-in routines for evaluating such functions in standard computing systems poses numerous challenges in numerical experiments and simulations. Motivated by the apparent need to circumvent these difficulties in performance analyses of cooperative wireless communications, this work presents an alternative method for obtaining the exact, approximate and asymptotic BFHF-free cumulative distribution function (CDF) of the end-to-end (e2e) signal-to-noise ratio (SNR) of multihop amplify-and-forward (AF) relaying wireless communication systems. As an illustration, the e2e performance analysis of an all-optical dual-hop free-space optical (FSO) transmission system over Gamma-Gamma turbulence in the presence of pointing errors is revisited. Specifically, new mathematical formulae for the statistical characteristics of the e2e SNR for systems with AF fixed-gain relaying as well as channel-state-information(CSI)assisted using heterodyne detection (HD) or intensity modulation with direct detection (IM/DD) are derived in terms of mathematically malleable and uniformly convergent infinite series of weighted Meijer G-functions. The usefulness of the derived CDFs is illustrated through derivation of traditional system performance metrics. The accuracy of the derived analytical formulae is verified via Monte Carlo simulations in MATLAB ${ }^{\circledR}$. Finally, based on results observed in this paper, useful expansions of common BFHFs in terms of easily computable univariate hypergeometric functions are proposed.
\end{abstract}

Index Terms-Gamma-Gamma distribution, pointing errors, bit error rate, outage probability, channel capacity, free-space optics, Fox H-function, Meijer G-function.

\section{INTRODUCTION}

Free-space optical (FSO) wireless communication has been hailed as an eminent alternative solution to optical fiber and radio frequency (RF) communication due to its costeffectiveness, ability to establish full-duplex high-speed wireless links over considerable distances, ease of deployment, energy efficiency, wider bandwidth as well as its operation on unlicensed spectrum, [1]-[4]. For these reasons, FSO links are a promising technology in space-based laser communications for backhauling large amounts of data from satellites to earth [1]. FSO systems use short optical light wavelengths that enable higher data transmission rates as well as narrow beam

T. R. Rasethuntsa is with the Department of Electrical \& Computer Engineering at McMaster University, Hamilton, ON, L8S 4L8, Canada (email: rasethut@mcmaster.ca). I. S. Ansari is with the James Watt School of Engineering, University of Glasgow, Glasgow G12 8QQ, U.K. (e-mail: imran.ansari@glasgow.ac.uk). width. This increases the difficulty of signal interception and thus offers more security as compared to RF systems. Despite all of the aforementioned desirable qualities, FSO technology is marred by atmospheric conditions such as rain, snow, fog and turbulence-induced irradiance fluctuations similar to fading experienced by RF technology [5]. Even in clear weather conditions, FSO channels will experience scintillation and these can adversely affect the quality of the FSO propagation channel [6]. Furthermore, FSO systems suffer from the misalignment between transmitter and receiver caused by small earthquakes, thermal expansion and winds among others, and these are known as pointing errors [7], [8]. Therefore, the effects of pointing errors as well as atmospheric turbulence need to be accurately characterized in statistical models and taken into account during system design and performance evaluation of FSO systems.

Driven by the need for statistical models that better characterize fading, shadowing and atmospheric turbulence-induced fluctuations, the past few decades have seen a rise in the interest to develop more flexible statistical distributions. This is often achieved by adding more parameters to already existing models [9]-[14]. One such model is the GammaGamma distribution [6] which encapsulates properties of the $K$-distribution [15] and the log-normal distribution [16], [17] originally developed to model strong and weak turbulence conditions, respectively. Yet, with improvement in the models that characterize various channel interference phenomena, arise corresponding increase in mathematical complexities when attempting to derive statistics of the end-to-end (e2e) signalto-noise ratio (SNR) in mathematically malleable forms. This is particularly ubiquitous in studies of performance of the socalled dual-hop amplify-and-forward (AF) relaying wireless transmission systems over FSO channels [3], [18]-[22] in which a relay is deployed between the source (transmitter) and the destination as a way of mitigating the effects of atmospheric turbulence or in order to circumvent lack of direct transmission. The complexities are evidenced by the prevalence of the bivariate Fox H-function (BFHF) [23, Eqs 2.2.1-2.2.8], [24, Definition 2.7] in recent literature, [19], [20], [22], [25]-[27]. We single out the work by Zedini et al. [22] in which exact and asymptotic closed-form performance metrics of an all-optical half-duplex AF relaying dual-hop FSO system are derived in correct albeit very tedious forms involving the BFHF.

It is well-known that closed-form expressions or accurate and easily computable approximate forms of performance 
metrics for wireless communication systems are integral in the design of such systems since they are a better alternative to the often time-consuming Monte Carlo simulations. It is the opinion of the authors that for closed-form or approximate expressions to be useful, they should also be less cumbersome to enable efficient implementation in standard computing systems. However, despite its usefulness as a general function encapsulating most elementary and special functions, the cumbersomeness of the BFHF coupled with its unavailability in any of the latest versions of numerical computing systems such as MATLAB ${ }^{\circledR}$, MATHEMATICA $^{\circledR}$ and MAPLE ${ }^{\circledR}$, has presented seemingly insurmountable impediments to efficient numerical experiments in research.

Several authors proposed codes for computing the BFHF on different platforms. Based on optimization theory, Chergui et al. [28] proposed what is undoubtedly the most ambitious MATLAB ${ }^{\circledR}$ and C/MEX routine for computing the general multivariate Fox H-function in [24, Appendix A.1] with an automated contour calculator, while a Python code for the multivariate Fox H-function was given in [29]. For these codes and many others scattered in the literature [30]-[35], the method of evaluating the Fox $\mathrm{H}$-function uses direct numerical integration of the corresponding Mellin-Barnes contour integral definition. According to Mathai and Saxena [23], the value of the univariate Fox $\mathrm{H}$-function for a given set of suitable parameters does not depend on the choice of an eligible contour (from an infinite number available). Yet, in implementation of the aforementioned codes, a contour choice does not always lead to quick convergence or desirable values and manual readjustments of the contour may become necessary with steeper penalties in computation time paid for larger contour lengths, especially when there are empty parameter sets. Given these and other possible computational issues, it will take time for the BFHF to be officially implemented in standard packages as alluded by [35], especially since its univariate counterpart [24, Definition 1.1] is yet to be implemented as well.

In the meantime, to maneuver around these limitations posed by the use of BFHFs in e2e performance analyses of all-optical dual-hop AF relaying systems, the present work's objective is to try and reduce the complexity and tediousness of the mathematical formulae of performance metrics in [22] so as to enable an easier implementation and evaluation in standard computing systems. Specifically, based on the pioneering work of Meijer [36], a simple method for obtaining BFHF-free cumulative distribution function (CDF) and probability density function (PDF) of the e2e SNR under fixed and variable gain scenarios is proposed. The usefulness of the derived CDFs and PDFs are explored via the derivation of BFHF-free traditional performance metrics such as average bit error rate (BER), outage probability (OP) and channel capacity under different adaptive transmission schemes. The rest of the paper is organized as follows : In Section II, model and channel descriptions are presented. Section III-A proposes the first main results of the paper with the derivation of exact and asymptotic statistical properties of the e2e SNR under fixedgain relaying. Section IV covers the derivation of various traditional performance metrics of the system under study in various forms. In Section $\mathrm{V}$, a variable-gain relay system is now considered and the statistical characteristics of the e2e SNR are derived. Numerical experiments and simulations explore the accuracy of the proposed formulae in Section VI. Finally, Section VII concludes the paper.

\section{Model And Channels Description}

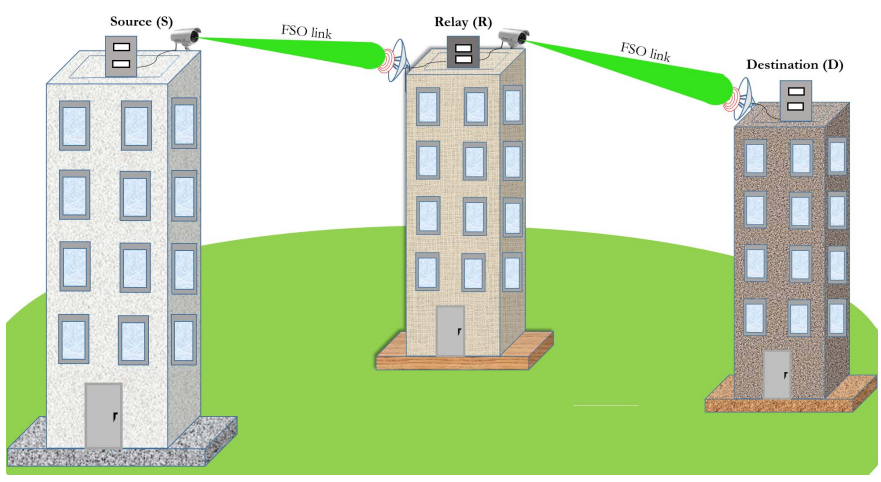

Figure 1: Illustration of the considered all-optical dual-hop FSO system

We consider a dual-hop relay FSO system as depicted on Figure 1 in which a source $(\mathrm{S})$ sends information to the destination (D) via a half-duplex AF relay node (R) equipped with receiver and transmitter apertures. Information is transmitted from $S$ to $R$ that amplifies the received signal and transmits it to terminal $D$. Furthermore, we suppose that the atmospheric turbulence of the $i$-th hop $(i=1,2)$ independently follows the Gamma-Gamma distribution $(\Gamma \Gamma)$ which is widely accepted as a suitable model for weak, moderate to strong turbulence conditions. Assuming that $S$ transmits a signal with an average power normalized to unity, the end-to-end (e2e) signal-tonoise-ratio (SNR) at D can be expressed for fixed-gain relay systems as [37]

$$
\gamma=\frac{\gamma_{1} \gamma_{2}}{\mathcal{G}+\gamma_{2}}
$$

where $\mathcal{G}$ is the fixed-gain at $\mathrm{R}, \gamma_{i}=\left(\eta_{e} I_{i}\right)_{i}^{\theta} / N_{o},(i=1,2)$, is the instantaneous SNR of the $i$-th hop, $\eta_{e}$ is the electrical-tooptical conversion ratio and $\theta_{i}$ denotes the detection method, i.e., $\theta_{i}=1$ corresponds to heterodyne detection (HD) and $\theta_{i}=2$ represents intensity modulation with direct detection (IM/DD) now commonly employed in practical applications. $I_{i}$ is the receiver irradiance and $N_{0}$ is the single-sided power spectral density of the additive white Gaussian noise (AWGN) at the $i$-th hop. The PDF of the instantaneous SNR of the $i$-th hop, denoted by $\gamma_{i}$, is given by [3]

$$
f_{\gamma_{i}}\left(\gamma_{i}\right)=\frac{\mathcal{T}_{i}}{\theta_{i} \gamma_{i}} G_{1,3}^{3,0}\left(\begin{array}{l|c}
\mathcal{K}_{i}\left(\gamma_{i}\right)^{\frac{1}{\theta_{i}}} & \xi_{i}^{2}+1 \\
\xi_{i}^{2}, \alpha_{i}, \beta_{i}
\end{array}\right)
$$

where $\mathcal{T}_{i}=\xi_{i}^{2} /\left(\Gamma\left(\alpha_{i}\right) \Gamma\left(\beta_{i}\right)\right), \mathcal{K}_{i}=h_{i} \alpha_{i} \beta_{i}\left(\mu_{i}\right)^{-\frac{1}{\theta_{i}}}, G(\cdot)$ is the Meijer G-function [38, Definition. 1] and $h_{i}=$ $\xi_{i}^{2} /\left(\xi_{i}^{2}+1\right) \cdot \xi_{i}^{2}=\frac{\omega_{z_{e q}, i}}{2 \sigma_{J, i}}$, with $\sigma_{J, i}$ as the jitter standard deviation at the receiver and $\omega_{z_{e q}, i}$ as the equivalent beam radius of the photo-detector for the receiver of the $i$-th hop. The average electrical SNR is given by $\mu_{i}=\mathbb{E}\left\langle\gamma_{i}\right\rangle=\bar{\gamma}_{i}$ for $\mathrm{HD}$ and $\mu_{i}=\bar{\gamma}_{i} \alpha \beta \xi_{i}^{2}\left(\xi_{i}^{2}+2\right) /\left[\left(\alpha_{i}+1\right)\left(\beta_{i}+1\right)\left(\xi_{i}^{2}+1\right)^{2}\right]$ 
for IM/DD. The parameters $\alpha_{i}$ and $\beta_{i}$ represent the effective number of large-scale and small-scale cells of the scattering process, respectively [6]. Since using maximum likelihood estimation for estimating the parameters $\alpha_{i}$ and $\beta_{i}$ or deriving their Cramér-Rao lower bound of their estimators is difficult to carry out, $\alpha_{i}$ and $\beta_{i}$ are usually calculated through the Rytov variance with the assumption of a plane wave propagation in the absence of inner scale and knowledge of link distance as well as refractive-index structure parameter [39], [40]. Consequently, $\alpha_{i}$ and $\beta_{i}$ cannot be chosen randomly in numerical experiments since they are related through the Rytov variance as follows [6]

$$
\begin{aligned}
& \alpha_{i}=\left\{\exp \left[\frac{0.49 \sigma_{R_{i}}^{2}}{\left(1+1.11 \sigma_{R_{i}}^{12 / 5}\right)^{7 / 6}}\right]-1\right\}^{-1}, \\
& \beta_{i}=\left\{\exp \left[\frac{0.51 \sigma_{R_{i}}^{2}}{\left(1+0.69 \sigma_{R_{i}}^{12 / 5}\right)^{5 / 6}}\right]-1\right\}^{-1}
\end{aligned}
$$

where $\sigma_{R_{i}}^{2}=1.23 C_{n, i}^{2}\left(\frac{2 \pi}{\lambda_{i}}\right)^{\frac{7}{6}} L_{i}^{\frac{11}{6}}$ denotes the Rytov variance with $\lambda_{i}, L_{i}$ and $C_{n, i}^{2}$ representing the wavelength, propagation distance and refractive-index structure of the laser light of the $i$-th hop, respectively. Furthermore, $\alpha_{i}>\beta_{i}$ and $\beta_{i}$ is lower bounded by 0.91398 as the Rytov variance $\sigma_{R_{i}} \rightarrow \infty$. In experimentation, optical turbulence can be varied as follows : for weak turbulence $\sigma_{R_{i}}^{2} \leq 0.3$. For moderate turbulence $0.3<\sigma_{R_{i}}^{2} \leq 5$ and for strong turbulence, $\sigma_{R_{i}}^{2}>5$. However, these are not standards and different values may be chosen, see [41]. In the present work, we use the definition of the Meijer G-function given in [38, Definition 1] as the Mellin-Barnes contour integral

$$
\begin{aligned}
& G_{p, q}^{m, n}\left(x \mid \begin{array}{c}
a_{1}, \ldots, a_{p} \\
b_{1}, \ldots, b_{q}
\end{array}\right) \\
& =\frac{1}{2 \pi i} \int_{\mathcal{L}} \frac{\prod_{j=0}^{m} \Gamma\left(b_{j}-s\right) \prod_{j=1}^{n} \Gamma\left(1-a_{j}+s\right) x^{s} d s}{\prod_{j=m+1}^{q} \Gamma\left(1-b_{j}+s\right) \prod_{j=n+1}^{p} \Gamma\left(a_{j}-s\right)}
\end{aligned}
$$

satisfying specific conditions of convergence. Using [42, Eqs. 1.16.4(1) and 8.2.2(40)], the CDF of $\gamma_{1}$ can be expressed as

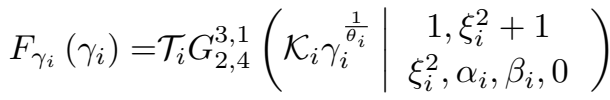

$$
\begin{aligned}
& =\frac{\theta_{i}^{\alpha_{i}+\beta_{i}-2} \mathcal{T}_{i}}{(2 \pi)^{\theta_{i}-1}} G_{2 \theta_{i}, 4 \theta_{i}}^{3 \theta_{i}, \theta_{i}}\left(\begin{array}{l|l}
\mathcal{K}_{i}^{\theta_{i}} \gamma_{i} & \begin{array}{c}
\Delta\left(\theta_{i}, 1\right), \mathbf{\Delta}_{i} \\
\theta_{i}^{2 \theta_{i}}
\end{array} \\
\mathbf{\nabla}_{i}, \Delta\left(\theta_{i}, 0\right)
\end{array}\right)
\end{aligned}
$$

where we have set $\boldsymbol{\Delta}_{i} \equiv \Delta\left(\theta_{i}, \xi_{i}^{2}+1\right)$ and also $\boldsymbol{\nabla}_{i} \equiv$ $\Delta\left(\theta_{i}, \xi_{i}^{2}\right), \Delta\left(\theta_{i}, \alpha_{i}\right), \Delta\left(\theta_{i}, \beta_{i}\right), \Delta\left(\theta_{i}, 0\right)$ for $i=1,2$ with $\Delta(k, \theta)=\left(\frac{\theta}{k}, \ldots, \frac{\theta+k-1}{k}\right)$ as a vector comprising $k$ terms. Recalling the relations $F_{\gamma_{1}}\left(\gamma_{1}\right)=\int_{0}^{\gamma_{1}} f_{\gamma_{1}}(x) d x=1-$ $\int_{\gamma_{1}}^{\infty} f_{\gamma_{1}}(x) d x$, we employ [42, Eq. 2.24.2(3)] to obtain the CDF of $\gamma$ in two more alternative forms as

$$
\begin{aligned}
F_{\gamma_{i}}\left(\gamma_{i}\right) & =1-\mathcal{T}_{i} G_{2,4}^{4,0}\left(\mathcal{K}_{i} \gamma_{i}^{\frac{1}{\theta_{i}}} \mid \begin{array}{c}
1, \xi_{i}^{2}+1 \\
\xi_{i}^{2}, \alpha_{i}, \beta_{i}, 0
\end{array}\right) \\
& =1-\frac{\theta_{i}^{\alpha_{i}+\beta_{i}-2} \mathcal{T}_{i}}{(2 \pi)^{\theta_{i}-1}} G_{2 \theta_{i}, 4 \theta_{i}}^{4 \theta_{i}, 0}\left(\begin{array}{l|l}
\mathcal{K}_{i}^{\theta_{i}} \gamma_{i} & \Delta\left(\theta_{i}, 1\right), \mathbf{\Delta}_{i} \\
\theta_{i}^{2 \theta_{i}} & \left.\begin{array}{c}
\mathbf{\nabla}_{i}, \Delta\left(\theta_{i}, 0\right)
\end{array}\right)
\end{array}\right)
\end{aligned}
$$

\section{Systems With FIXED-GAIN RELAYS}

\section{A. Statistical Properties}

In this section, we set out to derive the exact statistical properties of the e2e SNR for fixed-gain relay systems. We present the exact CDF, PDF, MGF as well as generalized moments of the e2e SNR.

\section{1) $C D F$ and PDF of end-to-end SNR:}

Theorem 1. The CDF and PDF of the e2e SNR for an alloptical dual-hop fixed-gain AF relaying system over GammaGamma turbulence in the presence of pointing errors is given in three different forms as shown in Table I. Moreover, all the series are uniformly convergent.

Proof: Please refer to Appendix A.

Remark 1. It is useful to have the CDF of $\gamma$ expressed in the three forms displayed in Table I when deriving various statistical characteristics and system performance metrics. In particular, it is observed that the form (11) is preferred over (8) when deriving an expression for channel capacity under optimal power and rate adaptation (see Section $I V-B 1)$ since it yields formulae that converge faster, while the form (13) is suitable for asymptotic analysis in Section III-B.

A useful observation from Theorem 1 is that the well-known method used in [22] would yield the CDF of $\gamma$ in terms the BFHF as

$$
\begin{aligned}
F_{\gamma}(\gamma)= & \prod_{i=1}^{2}\left(\frac{\theta_{i}^{\alpha_{i}+\beta_{i}-1} \mathcal{T}_{i}}{(2 \pi)^{\theta_{i}-1}}\right) \\
& \times G_{1,0: 2 \theta_{1}, 4 \theta_{2}+1 ; 4 \theta_{1}, 2 \theta_{1}+1}^{0,1: 3 \theta_{2}+1, \theta_{2} ; \theta_{1}, 3 \theta_{1}}\left(\begin{array}{c|c}
\frac{\mathcal{G} \mathcal{K}_{2}^{\theta_{2}}}{\theta_{2}^{\theta_{2}}} & \boldsymbol{\nabla}^{(1)} \\
\frac{\theta_{1}^{2 \theta_{1}}}{\mathcal{K}_{1}^{\theta_{1}} \gamma} & \mathbf{\Delta}^{(1)}
\end{array}\right)
\end{aligned}
$$

where $\boldsymbol{\nabla}^{(1)} \equiv 1: \Delta\left(\theta_{2}, 1\right), \mathbf{\Delta}_{2} ; 1-\boldsymbol{\nabla}_{1}, 1-\Delta\left(\theta_{1}, 0\right), \mathbf{\Delta}^{(1)} \equiv$ - : $0, \boldsymbol{\nabla}_{2}, \Delta\left(\theta_{2}, 0\right) ; 1-\Delta\left(\theta_{1}, 1\right), 1,1-\boldsymbol{\Delta}_{1}$, and $G . \cdot ; \cdot(\cdot)$ denotes the bivariate Meijer G-function (BMGF) which is a special case of the bivariate Fox $\mathrm{H}$-function with the third characteristic [44, Chap. 2], [24, Def. 2.7]. Furthermore, Eq. (15) will always converge according to [44, Consequence 13.1]. 
$\mathrm{H}$

Table I: Different forms of the CDF and PDF of $\gamma$ for FixedGain Relay Systems Based on Meijer [43, Theorem 2, Eqs. 33]

$$
\begin{aligned}
F_{\gamma}(\gamma)= & \frac{\theta_{1}^{\alpha_{1}+\beta_{1}-2} \mathcal{T}_{1}}{(2 \pi)^{\theta_{1}-1}} \sum_{p=0}^{\infty} \frac{\mathcal{A}(p)}{p !} \\
& \times G_{2 \theta_{1}+1,4 \theta_{1}+1}^{3 \theta_{1}, \theta_{1}+1}\left(\begin{array}{c|c}
\frac{\mathcal{K}_{1}^{\theta_{1}} \gamma}{\theta_{1}^{2 \theta_{1}}} & \left.\begin{array}{c}
1-p, \Delta\left(\theta_{1}, 1\right), \mathbf{\Delta}_{1} \\
\mathbf{\nabla}_{1}, \Delta\left(\theta_{1}, 0\right), 1
\end{array}\right)
\end{array}\right.
\end{aligned}
$$

where $\mathcal{A}(0)=1$ and for $p \geq 1$,

$$
\begin{aligned}
\mathcal{A}(p) & =\frac{\theta_{2}^{\alpha_{2}+\beta_{2}-2} \mathcal{T}_{2}}{(2 \pi)^{\theta_{2}-1} \Gamma(p)} G_{\theta_{2}+1,3 \theta_{2}+1}^{3 \theta_{2}+1,1}\left(\frac{\mathcal{G} \mathcal{K}_{2}^{\theta_{2}}}{\theta_{2}^{2 \theta_{2}}} \mid \begin{array}{c}
1, \mathbf{\Delta}_{2} \\
p, \mathbf{\nabla}_{2}
\end{array}\right) \\
f_{\gamma}(\gamma) & =\frac{\theta_{1}^{\alpha_{1}+\beta_{1}-2} \mathcal{T}_{1}}{(2 \pi)^{\theta_{1}-1}} \sum_{p=0}^{\infty} \frac{\mathcal{A}(p)}{p !} \\
& \times \frac{1}{\gamma} G_{2 \theta_{1}+2,4 \theta_{1}+2}^{3 \theta_{1}, \theta_{1}+2}\left(\begin{array}{c|c}
\frac{\mathcal{K}_{1}^{\theta_{1}} \gamma}{\theta_{1}^{2 \theta_{1}}} & 0,1-p, \Delta\left(\theta_{1}, 1\right), \mathbf{\Delta}_{1} \\
\mathbf{\nabla}_{1}, \Delta\left(\theta_{1}, 0\right), 1,1
\end{array}\right)
\end{aligned}
$$

$$
\begin{aligned}
& F_{\gamma}(\gamma)=1-\frac{\theta_{1}^{\alpha_{1}+\beta_{1}-2} \mathcal{T}_{1}}{(2 \pi)^{\theta_{1}-1}} \sum_{p=0}^{\infty} \frac{(-1)^{p} \mathcal{A}(p)}{p !} \\
& \times G_{2 \theta_{1}+1,4 \theta_{1}+1}^{4 \theta_{1}+1,0}\left(\begin{array}{c|c}
\mathcal{K}_{1}^{\theta_{1}} \gamma & 1-p, \Delta\left(\theta_{1}, 1\right), \mathbf{\Delta}_{1} \\
\hline \theta_{1}^{2 \theta_{1}} & \mathbf{\nabla}_{1}, \Delta\left(\theta_{1}, 0\right), 1
\end{array}\right) \\
& f_{\gamma}(\gamma)=\frac{\theta_{1}^{\alpha_{1}+\beta_{1}-2} \mathcal{T}_{1}}{(2 \pi)^{\theta_{1}-1}} \sum_{p=0}^{\infty} \frac{(-1)^{p} \mathcal{A}(p)}{p !} \\
& \times \frac{1}{\gamma} G_{2 \theta_{1}+2,4 \theta_{1}+2}^{4 \theta_{1}+2,}\left(\begin{array}{c|c}
\mathcal{K}_{1}^{\theta_{1}} \gamma & \begin{array}{c}
1-p, \Delta\left(\theta_{1}, 1\right), \mathbf{\Delta}_{1}, 0 \\
\theta_{1}^{2 \theta_{1}}
\end{array} \\
1, \nabla_{1}, \Delta\left(\theta_{1}, 0\right), 1
\end{array}\right)
\end{aligned}
$$

$F_{\gamma}(\gamma)=\theta_{1} \mathcal{T}_{1} \sum_{p=0}^{\infty} \frac{\mathcal{A}^{*}(p)}{p !} G_{2,4}^{3,1}\left(\begin{array}{l|l}\mathcal{K}_{1} \gamma^{\frac{1}{\theta_{1}}} & \begin{array}{c}1-p, \xi_{1}^{2}+1 \\ \xi_{1}^{2}, \alpha_{1}, \beta_{1}, 0\end{array}\end{array}\right)$,

$\mathcal{A}^{*}(0)=1$ and for $p \geq 1, \mathcal{A}^{*}(p)=\frac{\theta_{2}^{\alpha_{2}+\beta_{2}-2} \mathcal{T}_{2}}{(2 \pi)^{\theta_{2}-1}} \sum_{q=0}^{p} \mathcal{I}(q)$,

$$
\mathcal{I}(q)=\frac{(-1)^{q}\left(\begin{array}{l}
p \\
q
\end{array}\right)}{\Gamma\left(\frac{q}{\theta_{1}}\right)} G_{\theta_{2}+1,3 \theta_{2}+1}^{3 \theta_{2}+1,1}\left(\begin{array}{c|c}
\mathcal{G K}_{2}^{\theta_{2}} & 1-\frac{q}{\theta_{1}}, \mathbf{\Delta}_{2} \\
\theta_{2}^{2 \theta_{2}} & 0, \mathbf{\nabla}_{2}
\end{array}\right)
$$

Corollary 1. For real $x \neq 0, y \neq 0, \boldsymbol{a}, \boldsymbol{b}, \boldsymbol{c}, \boldsymbol{d}, \boldsymbol{e}$, and $\boldsymbol{f}$, the following result can be derived from Eq. (8)

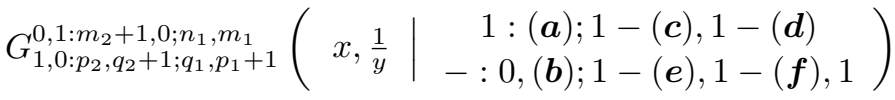

$$
\begin{aligned}
& =\sum_{k=1}^{\infty} \frac{1}{k ! \Gamma(k)} G_{p_{2}+1, q_{2}+1}^{m_{2}+1,1}\left(x \mid \begin{array}{l}
1,(\boldsymbol{a}) \\
k,(\boldsymbol{b})
\end{array}\right) \\
& \times G_{p_{1}+1, q_{1}+1}^{m_{1}, n_{1}+1}\left(y \mid \begin{array}{c}
1-k,(\boldsymbol{e}),(\boldsymbol{f}) \\
(\boldsymbol{c}),(\boldsymbol{d}), 1
\end{array}\right)
\end{aligned}
$$

Proof: Expanding the univariate Meijer G-functions according to (5) and interchanging the summation and integration signs, we get an inner sum over $k$ as

$$
\begin{aligned}
\mathcal{S}=\sum_{k=1}^{\infty} \frac{\Gamma(k-s) \Gamma(k-t)}{\Gamma(k) \Gamma(k+1)} & =\sum_{k=0}^{\infty} \frac{\Gamma(1+k-s) \Gamma(1+k-t)}{\Gamma(k+1) \Gamma(k+2)} \\
& =\Gamma(1-s) \sum_{k=0}^{\infty} \frac{(1-s)_{k}(1-t)_{k}}{\Gamma(1-t)(2)_{k} k !}
\end{aligned}
$$

where we have set $k=k+1$ and used the Pochhammer symbol defined by $(a)_{n}=\Gamma(a+n) / \Gamma(a)$. Next, application of [42, Definition 7.2.3(1)] and [42, Eq. 7.3.5(2)] yield

$$
\begin{aligned}
\mathcal{S} & =\Gamma(1-s) \Gamma(1-t){ }_{2} F_{1}(1-s, 1-t ; 2 ; 1) \\
& =\frac{\Gamma(1-s) \Gamma(1-t) \Gamma(s+t)}{\Gamma(1+s) \Gamma(1+t)}, \Re(s+t)>0
\end{aligned}
$$

Therefore, collecting all Gamma function terms under the double contour integral, we employ [24, Def. 2.7] to obtain the desired BMGF expression in Eq. (16).

A plot of PDF and CDF curves of $\gamma$ based on (10) and (8) under moderate turbulence are displayed on Figs 2 and 3, where we have employed the MATLAB ${ }^{\circledR}$ code proposed by [45] to generate $10^{7} \Gamma \Gamma$ random variables for each curve. In

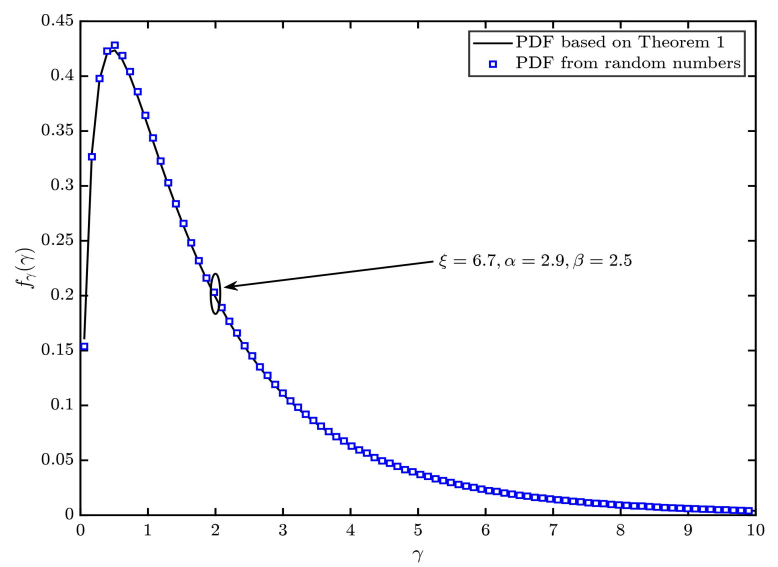

Figure 2: Analytical PDF against the simulated version under HD based on 25 terms of (10) with average electrical SNR per hop being $5 \mathrm{~dB}$.

Table II at the top of the next page, the behaviour of the CDF in Eq. (8) is demonstrated for weak turbulence. It is evident the convergence rate of the series is influenced by the size of the value being estimated, with some arguments leading to slower convergence rates. Overall, (8) appears to be accurate to at least 3 decimal places for a truncation number $P=25$ and improves, albeit very slowly due to the oscillatory nature of the convergence, as $P$ gets larger.

2) Generalized moments of end-to-end SNR: The generalized moments of the end-to-end SNR $\gamma$ make possible the investigation of the inherent statistical properties of $\gamma$ such as mean, variance, kurtosis and skewness among others. Furthermore, the $n$-th order moment of $\gamma$ is useful for obtaining $n$-th order amount of fading. The $n$-th order moment of $\gamma$ is 
Table II: Comparison of Exact CDF of $\gamma$ and CDF from Theorem 1 for $(\xi, \alpha, \beta)=(6.7,2.902,2.51)$

\begin{tabular}{lcccc}
\hline$(\bar{\gamma}, \gamma)$ & Truncation Number $P$ & Exact Value & $\begin{array}{c}\text { Value Based on Theorem 1 Eq. } \\
(8)\end{array}$ & Absolute Error \\
\hline$(0 \mathrm{~dB}, 0.96)$ & & & 0.900400 & 0.000390 \\
& 25 & 0.900010 & 0.900131 & 0.000121 \\
& 50 & & 0.900096 & 0.000086 \\
\hline 100 & & 0.900037 & 0.000027 \\
\hline 150 & 0.500010 & 0.499896 & 0.000114 \\
& 25 & 0.500071 & 0.000061 \\
& 50 & & 0.499988 & 0.000002 \\
& 100 & & 0.500021 & 0.000001 \\
\hline$(10 \mathrm{~dB}, 2.54)$ & 150 & 0.199985 & 0.000095 \\
& 50 & 0.200080 & 0.199957 & 0.000019 \\
\hline
\end{tabular}

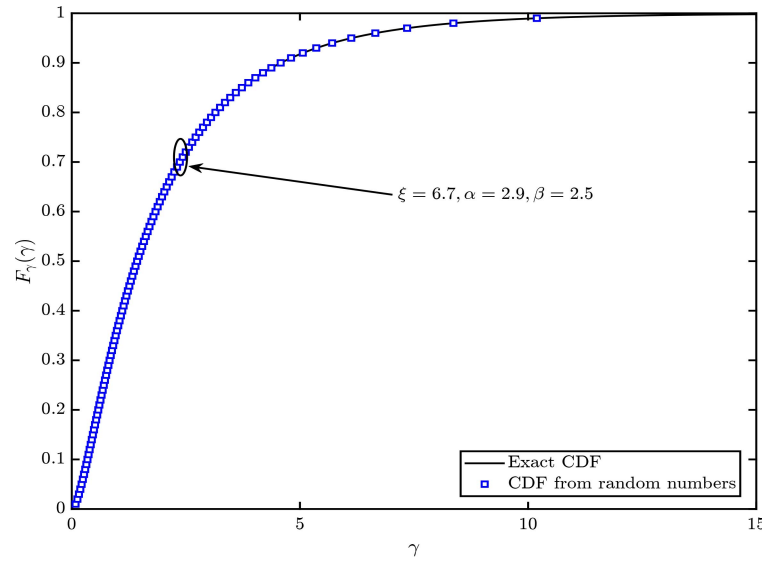

Figure 3: Analytical CDF against the simulated version under HD based on 25 terms of (8) with average electrical SNR per hop being $5 \mathrm{~dB}$.

derived as for $n \geq 2$ as

$$
\begin{aligned}
& \mathbb{E}\left\langle\gamma^{n}\right\rangle=\frac{n \theta_{1}^{\alpha_{1}+\beta_{1}-2} \mathcal{T}_{1} \theta_{1}^{2 n \theta_{1}}}{(2 \pi)^{\theta_{1}-1} \mathcal{K}_{1}^{n \theta_{1}}} \frac{\Gamma\left(\mathbf{\nabla}_{1}^{\prime}+n\right)}{\Gamma\left(\mathbf{\Lambda}_{1}^{\prime}+n\right)}\left[1+\frac{\theta_{2}^{\alpha_{2}+\beta_{2}-2} \mathcal{T}_{2}}{\Gamma(n)(2 \pi)^{\theta_{2}-1}}\right. \\
& \left.\times G_{\theta_{2}+2,3 \theta_{2}+2}^{3 \theta_{2}+1,2}\left(\begin{array}{c|c}
\mathcal{G K}_{2}^{\theta_{2}} & 1-n, 1, \mathbf{\Delta}_{2} \\
\hline \theta_{2}^{2 \theta_{2}} & 1, \mathbf{\nabla}_{2}, 0
\end{array}\right)\right]
\end{aligned}
$$

where $\boldsymbol{\Delta}_{1}^{\prime} \equiv \Delta\left(\theta_{1}, 1\right), \boldsymbol{\Lambda}_{1}$ and $\boldsymbol{\nabla}_{1}^{\prime} \equiv \boldsymbol{\nabla}_{1}, \Delta\left(\theta_{1}, 0\right)$ such that if $\boldsymbol{A}=\left[a_{1}, \ldots, a_{m}\right]$, then $\Gamma(\boldsymbol{A})=\prod_{i=1}^{m} \Gamma\left(a_{i}\right)$. The proof of (19) follows the proof of Corollary 1 by using (11) and the Mellin Transform in $\mathbb{E}\left\langle\gamma^{n}\right\rangle=n \int_{0}^{\infty} \gamma^{n-1}\left[1-F_{\gamma}(\gamma)\right] d \gamma$.

3) Moment generating function of end-to-end SNR: The moment generating function (MGF) is another useful statistical characteristic which can be used to generate $n$-th order moments and to also obtain the BER of $\gamma$ under non-coherent modulation. The MGF of $\gamma$ is defined as

$$
\mathcal{M}_{\gamma}(s)=s \int_{0}^{\infty} e^{-s \gamma} F_{\gamma}(\gamma) d \gamma
$$

Therefore, substituting (8), individually into (20) followed by the use of [42, Eq. 2.24.3(1)], we have that the MGF of $\gamma$ can be written as

$$
\begin{aligned}
\mathcal{M}_{\gamma}(s)= & \frac{\theta_{1}^{\alpha_{1}+\beta_{1}-2} \mathcal{T}_{1}}{(2 \pi)^{\theta_{1}-1}} \sum_{p=0}^{\infty} \frac{\mathcal{A}(p)}{p !} \\
& \times G_{2 \theta_{1}+2,4 \theta_{1}+1}^{3 \theta_{1}, \theta_{1}+2}\left(\begin{array}{c|c}
\mathcal{K}_{1}^{\theta_{1}} & 0,1-p, \Delta\left(\theta_{1}, 1\right), \mathbf{\Lambda}_{1} \\
s \theta_{1}^{2 \theta_{1}} & \mathbf{\nabla}_{1}, \Delta\left(\theta_{1}, 0\right), 1
\end{array}\right)
\end{aligned}
$$

Obviously, we may obtain another form for the MGF of $\gamma$ using (11). We will avoid stating these alternative forms for the sake of brevity.

\section{B. Asymptotic Statistical Properties of e2e SNR}

1) Asymptotic CDF of e2e SNR for $\mu_{2} \rightarrow \infty$ : In this section, we seek to derive the asymptotic CDF of $\gamma$ when the average electrical SNR of the R-D link $\mu_{2}$ is asymptotically large. In order to achieve this, we apply [42, Eq. 8.2.2(3)] to $\mathcal{A}(p)$ in (13) and recall that the generalized hypergeometric function [42, Eq. 7.2.3(1)] has the property that ${ }_{p} F_{q}\left[(\cdot)_{p} ;(\cdot)_{q} ; 0\right]=1[42$, Eq. 7.2.3(3)] so that we obtain the asymptotic form of $\mathcal{I}(q)$ from (13) which is independent of $q$ as

$$
\mathcal{I}(q) \dot{\sim} \frac{\theta_{2}^{\alpha_{2}+\beta_{2}-2}}{(2 \pi)^{\theta_{2}-1}} \frac{\Gamma\left(\mathbf{\Lambda}_{2}\right)}{\Gamma\left(\mathbf{v}_{2}\right)}
$$

It follows that $\sum_{p=1}^{\infty} \sum_{q=0}^{p}(-1)^{q}\left(\begin{array}{c}p \\ q\end{array}\right) \mathcal{I}(q)=0$ and therefore, the asymptotic CDF of the e2e SNR as $\mu_{2} \rightarrow \infty$ is equivalent to the leading term (i.e. series term at $p=0$ ) of (8), (11) and (13) which is simply the CDF of the S-R link SNR $\gamma_{1}$. That is,

$$
F_{\gamma}(\gamma) \dot{\sim} \theta_{1} \mathcal{T}_{1} G_{2,4}^{3,1}\left(\begin{array}{l|c}
\mathcal{K}_{1} \gamma^{\frac{1}{\theta_{1}}} & 1, \xi_{1}^{2}+1 \\
\xi_{1}^{2}, \alpha_{1}, \beta_{1}, 0
\end{array}\right)
$$

Following the same procedure as in the case when $\mu_{2} \rightarrow \infty$, we can show that the asymptotic CDF of $\gamma$ as $\mu_{1} \rightarrow \infty$ is also given by (23).

2) Asymptotic MGF of e2e SNR: The asymptotic CDF in (23) can be used for deriving asymptotic MGF of $\gamma$ by simply setting $p=0$ in (21) so that we get

$\mathcal{M}_{\gamma}(s) \dot{\sim} \frac{\theta_{1}^{\alpha_{1}+\beta_{1}} \mathcal{T}_{1}}{\theta_{1}(2 \pi)^{\theta_{1}-1}} G_{2 \theta_{1}+1,4 \theta_{1}}^{3 \theta_{1}, \theta_{1}+1}\left(\begin{array}{c|c}\frac{\mathcal{K}_{1}^{\theta_{1}}}{s \theta_{1}^{2 \theta_{1}}} & \begin{array}{c}0, \Delta\left(\theta_{1}, 1\right), \mathbf{\Delta}_{1} \\ \mathbf{\nabla}_{1}, \Delta\left(\theta_{1}, 0\right)\end{array}\end{array}\right)$ 
IV. Applications to the Performance Evaluation OF FIXED-GAIN DUAL-HOP FSO TRANSMISSION SYSTEMS

Channel capacity is an invaluable performance metric in the design of wireless communication systems since it serves as an upper limit on the transmission rate for reliable communications [46]-[48]. This performance metric has been studied over various communication scenarios and channel interference models using different adaptive transmission schemes [47][51]. However, in the case of dual-hop AF-relay-assisted wireless communication systems, the increased mathematical complexity in the forms of the CDF of the e2e SNR presents great computational and mathematical difficulties in deriving e2e channel capacity under different adaptive transmission techniques. This is particularly true for dual-hop FSO and mixed RF/FSO transmission systems with existing works such as [26] and [27] indicating how cumbersome the mathematical expressions for channel capacity using the OPRA policy are. In this section, we demonstrate what is undoubtedly the most attractive feature of the proposed CDF forms from Table I in simplifying the mathematical hurdles involved in deriving and computing effective capacity and channel capacity under ORA, OPRA, and TIFR adaptive transmission policies in forms which can easily be implemented in standard computing systems. In this work, we will only focus on HD for effective capacity as well as the OPRA and the TIFR policies.

\section{A. Channel capacity with receiver CSI}

We will first consider schemes with full receiver CSI, i.e. ergodic capacity (ORA) and effective capacity.

1) Optimal rate adaptation: The optimal rate adaptation (ORA) transmission policy assumes a constant transmit power is maintained. Under this scheme, also commonly known as ergodic capacity, the channel capacity of the e2e SNR is defined as [3]

$$
\mathbb{C}_{O}=\frac{1}{2} \mathbb{E}\left\langle\log _{2}(1+\varphi \gamma)\right\rangle=\frac{1}{\ln (4)} \int_{0}^{\infty} \ln (1+\varphi \gamma) f_{\gamma}(\gamma) d \gamma
$$

where $\varphi=e /(2 \pi)$ and $\varphi=1$ for IM/DD and HD, respectively. Note that $\varphi=e /(2 \pi)$ corresponds the value of $\varphi$ for a lower bound of $\mathbb{C}_{O}$ in the absence of peak-power constraints while the capacity is exact for HD [52, Theorem 6], [22]. Expressing $\ln (1+\varphi \gamma)$ in terms of the Meijer G-function using [42, Eq. 8.4.6(5)] and substituting this together with (10) into (25), we apply [42, Eq. 2.24.1(1)] to obtain

$$
\begin{aligned}
\mathbb{C}_{O} & =\frac{\theta_{1}^{\alpha_{1}+\beta_{1}-2} \mathcal{T}_{1}}{\ln (4)(2 \pi)^{\theta-1}} \sum_{p=0}^{\infty} \frac{\mathcal{A}(p)}{p !} \\
& \times G_{2 \theta_{1}+4,4 \theta_{1}+4}^{3 \theta_{1}+2, \theta_{1}+3}\left(\begin{array}{c|c}
\mathcal{K}_{1}^{\theta_{1}} & 0,1-p, \Delta\left(\theta_{1}, 1\right), 0,1, \mathbf{\Lambda}_{1} \\
\varphi \theta_{1}^{2 \theta_{1}} & \mathbf{\nabla}_{1}, 0,0, \Delta\left(\theta_{1}, 0\right), 1,1
\end{array}\right)
\end{aligned}
$$

The asymptotic ORA capacity is obtained by substituting $p=$ 0 into (26) to yield the asymptotic $\mathbb{C}_{O}$ as follows

$$
\frac{\theta_{1}^{\alpha_{1}+\beta_{1}-2} \mathcal{T}_{1}}{\ln (4)(2 \pi)^{\theta-1}} G_{2 \theta_{1}+3,4 \theta_{1}+3}^{3 \theta_{1}+2, \theta_{1}+2}\left(\begin{array}{c|c}
\mathcal{K}_{1}^{\theta_{1}} & 0, \Delta\left(\theta_{1}, 1\right), 0,1, \mathbf{\Delta}_{1} \\
\hline \varphi \theta_{1}^{2 \theta_{1}} & \mathbf{\nabla}_{1}, 0,0, \Delta\left(\theta_{1}, 0\right), 1
\end{array}\right)
$$

2) Effective capacity under heterodyne detection: Next generation wireless networks aim at supporting a host of quality-of-service (QoS) requirements and in order to assess the performance of these systems with QoS metrics such as delay, data rate and delay-violation probability, $\mathrm{Wu}$ and Rohit [53] introduced the concept of effective capacity (EC). EC, which has been studied for AF relay-assisted wireless networks in [54]-[56], is defined as the maximum constant arrival rate that a wireless channel can support while a given QoS requirement is guaranteed [54]. Mathematically, EC is defined for half-duplex dual-hop AF relay systems as [53], [55]

$$
\mathbb{C}_{E}=\frac{-1}{\phi B \boldsymbol{T}_{f}} \ln \left[\int_{0}^{\infty} f_{\gamma}(\gamma)(1+\gamma)^{-\frac{\phi B T_{f}}{2 \ln (2)}}\right]
$$

where $\phi, B$ and $\boldsymbol{T}_{f}$ denote the QoS exponent, system bandwidth and fading block/frame length, respectively. Small $\phi$ values correspond to slow decaying rate and looser QoS constraints while large $\phi$ represent fast decaying rate with more stringent QoS constraints [55]. Here, we are assuming the relay amplifies and re-transmits the received signal to the destination without any delays or buffering constraints [54]. Now, substituting (10) into (28) and employing [42, Eq. 2.24.2(4)], we get the integral in (28) as

$$
\mathcal{I}=\frac{\mathcal{T}_{1}}{\Gamma\left(\frac{\phi B \boldsymbol{T}_{f}}{\ln (4)}\right)} \sum_{p=0}^{\infty} \frac{\mathcal{A}(p)}{p !} G_{2,4}^{4,1}\left(\begin{array}{l|c}
\mathcal{K}_{1} & \begin{array}{c}
1-p, \xi_{1}^{2}+1 \\
\xi_{1}^{2}, \alpha_{1}, \beta_{1}, \frac{\phi B \boldsymbol{T}_{f}}{\ln (4)}
\end{array}
\end{array}\right)
$$

Therefore, putting (29) into (28) yields the e2e EC for a dual-hop system over gamma-gamma turbulence with pointing errors. The asymptotic EC is given by

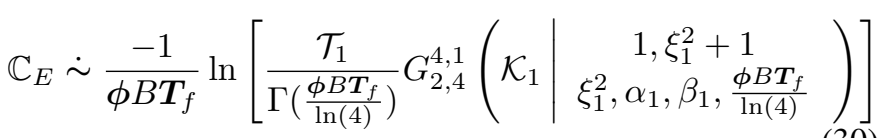

\section{B. Channel capacity with receiver and transmitter CSI}

1) Channel inversion with fixed rate under heterodyne detection: Another simple technique which offers easy implementation is to maintain a constant SNR at the receiver with fixed-rate modulation and fixed code design or channel inversion with fixed rate (CIFR) [57]. Under this technique, the channel capacity of the e2e SNR $\gamma$ is defined as [57]

$$
\mathbb{C}_{C}=\frac{B}{2 \ln 2} \ln \left(1+\frac{1}{\int_{0}^{\infty} \frac{1}{\gamma} f_{\gamma}(\gamma) d \gamma}\right)
$$

It is immediately obvious that (31) is not possible to compute since the Meijer G-function in (19) is undefined for $n=-1$ as per definition (5). Furthermore, the CIFR technique may suffer large capacity penalties in severe fading conditions as compared to other techniques. To circumvent this shortcoming, a modified inversion which inverts the channel fading above a predetermined truncated fade $\gamma_{o}$ (TIFR scheme) is often used. 
2) Truncated channel inversion with fixed rate under heterodyne detection: The channel capacity based on the truncated channel inversion with fixed rate (TIFR) policy is defined as [57]

$$
\left.\mathbb{C}_{T}=\frac{B}{2 \ln 2} \ln [1+\underbrace{\int_{\gamma_{0}}^{\infty} \frac{1}{\gamma} f_{\gamma}(\gamma) d \gamma}_{\mathcal{X}})^{-1}\right] \operatorname{Pr}\left(\gamma>\gamma_{o}\right)
$$

where (12) and [42, Eq. 2.24.2(3)] lead to exact and asymptotic forms

$$
\begin{aligned}
& \mathcal{X}=\mathcal{T}_{1} \sum_{p=0}^{\infty} \frac{(-1)^{p} \mathcal{A}(p)}{p !} G_{3,5}^{5,0}\left(\begin{array}{l|l}
\mathcal{K}_{1} \gamma_{o} & \begin{array}{c}
1-p, \xi_{1}^{2}+1,2 \\
1, \xi_{1}^{2}, \alpha_{1}, \beta_{1}, 1
\end{array}
\end{array}\right), \\
& \int_{\gamma_{0}}^{\infty} \frac{1}{\gamma} f_{\gamma}(\gamma) d \gamma \dot{\sim} \mathcal{T}_{1} G_{2,4}^{4,0}\left(\begin{array}{l|c}
\mathcal{K}_{1} \gamma_{o} & \xi_{1}^{2}+1,2 \\
1, \xi_{1}^{2}, \alpha_{1}, \beta_{1}
\end{array}\right) .
\end{aligned}
$$

Therefore, substituting (33) into (32), we obtain channel capacity under the TIFR scheme with $\operatorname{Pr}\left(\gamma>\gamma_{o}\right)=1-F_{\gamma}\left(\gamma_{o}\right)$, where $F_{\gamma}$ is as defined in (11) or (8). $\mathbb{C}_{C}$ can then be computed as the limit of $\mathbb{C}_{T}$ as $\gamma_{o} \rightarrow 0$, which from [42, Eqs. 8.2.2(3) and 7.2.3(3)], implies that $\mathbb{C}_{C} \rightarrow 0$.

3) Optimal power and rate adaptation under heterodyne detection: The optimal power and rate adaptation (OPRA) technique achieves the highest possible capacity with CSI by employing a multiplexed multiple code-book design in order to match the transmission power and rate of the system, [47]. The channel capacity for a system employing the ORA technique is defined as [57]

$$
\mathbb{C}_{P}=\frac{B}{2} \int_{\gamma_{o}}^{\infty} \log _{2}\left(\frac{\gamma}{\gamma_{o}}\right) f_{\gamma}(\gamma) d \gamma
$$

where $\gamma_{o}$ is the optimal cut-off SNR below which data transmission is not permitted. In order to ensure the latter, $\gamma_{o}$ must satisfy the relation

$$
\int_{\gamma_{o}}^{\infty}\left(\frac{1}{\gamma_{o}}-\frac{1}{\gamma}\right) f_{\gamma}(\gamma) d \gamma=1
$$

Using integration by parts, (35) may be expressed in terms of the CDF of $\gamma$ as [47]

$$
\mathbb{C}_{P}=\frac{B}{2 \ln 2} \int_{\gamma_{o}}^{\infty} \frac{1-F_{\gamma}(\gamma)}{\gamma} d \gamma \text {. }
$$

Therefore, substituting (11) into (37) and applying [42, Eq. 2.24.2(3)], we obtain

$$
\mathbb{C}_{P}=\frac{B \mathcal{T}_{1}}{\ln (4)} \sum_{p=0}^{\infty} \frac{\mathcal{A}(p)}{p !(-1)^{p}} G_{3,5}^{5,0}\left(\begin{array}{l|l}
\mathcal{K}_{1} \gamma_{o} & \begin{array}{c}
1-p, 1, \xi_{1}^{2}+1 \\
0, \xi_{1}^{2}, \alpha_{1}, \beta_{1}, 0
\end{array}
\end{array}\right)
$$

The asymptotic OPRA capacity is given by

$$
\mathbb{C}_{P} \dot{\sim} \frac{B \mathcal{T}_{1}}{\ln (4)} G_{3,5}^{5,0}\left(\begin{array}{l|c}
\mathcal{K}_{1} \gamma_{o} & 1,1, \xi_{1}^{2}+1 \\
0, \xi_{1}^{2}, \alpha_{1}, \beta_{1}, 0
\end{array}\right)
$$

It is also worth noting that the SNR cut-off relation in (36) can be re-written as

$$
\gamma_{o}=\int_{\gamma_{o}}^{\infty} \frac{\left(\gamma-\gamma_{o}\right)}{\gamma} f_{\gamma}(\gamma) d \gamma=g\left(\gamma_{o}\right)
$$

It was observed during numerical experiments that (40) can only be derived using (12) since the other PDF forms result in infeasible solution to (41). Therefore, the use of (12) and [42, Eq. 2.24.2(3)] in (40) yields

$g\left(\gamma_{o}\right)=\mathcal{T}_{1} \sum_{p=0}^{\infty} \frac{(-1)^{p} \mathcal{A}(p)}{p !} G_{3,5}^{5,0}\left(\begin{array}{l|l}\mathcal{K}_{1} \gamma_{o} & \begin{array}{c}1-p, \xi_{1}^{2}+1,2 \\ 0, \xi_{1}^{2}, \alpha_{1}, \beta_{1}, 1\end{array}\end{array}\right)$

Clearly, $\gamma_{o}$ is a fixed point of the non-linear equation $g\left(\gamma_{o}\right)=$ $\gamma_{o}$. According to Annamalai et al. [47], $\gamma_{o} \in(0,1]$ irrespective of the number of relays or channel model employed and this follows from the properties of a $\mathrm{CDF}$ of a random variable, see Figure 4. Therefore, $g\left(\gamma_{o}\right)=\gamma_{o}$ can be solved via iterative algorithms such as the Newton-Raphson method with a starting point conveniently selected from the interval $(0,1]$.

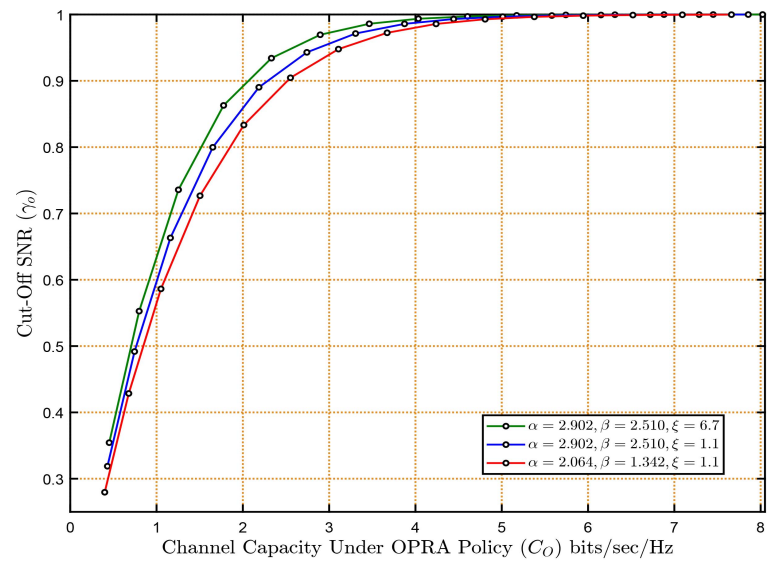

Figure 4: Typical $\gamma_{o}$ VS $\mathbb{C}_{P}$ curves under different turbulence conditions and pointing errors showing that $\gamma_{o}$ is constrained to the interval $(0,1]$ as suggested in [47]. Here, HD is employed and the average SNR per hop is being varied from $2 \mathrm{~dB}$ to $50 \mathrm{~dB}$ over 15 equidistant points for each curve.

\section{Outage probability}

Outage probability (OP) is defined as the probability that the e2e SNR does not exceed a predetermined threshold $\gamma_{t h}$. Mathematically, OP is expressed as

$$
P_{o p}=\operatorname{Pr}\left(\gamma<\gamma_{t h}\right)=F_{\gamma}\left(\gamma_{t h}\right)
$$

\section{Bit error rate probability}

For a number of modulation schemes, the average BER of the e2e SNR $\gamma$ can be written in terms of its CDF as [19]

$$
\bar{P}_{e}=\frac{\delta}{2 \Gamma(b)} \sum_{l=1}^{m} a_{l}^{b} \int_{0}^{\infty} \gamma^{b-1} e^{-a_{l} \gamma} F_{\gamma}(\gamma) d \gamma
$$

where the parameters $a$ and $b$ vary according to a modulation scheme. In particular, for $\delta=m=1, a_{l}=1$ and $b=1 / 2$, we obtain the coherent binary frequency shift keying (BFSK) modulation under heterodyne detection. When $\delta=m=1$, and $a_{l}=b=1 / 2$, we obtain on-off keying (OOK) modulation for IM/DD. The reader is referred to [19, Table I] for parameter choices in the case of M-PSK and M-QAM modulation schemes under heterodyne detection. Now, substituting (8) into 
(43) and using [42, Eq. 2.24.3(1)], we obtain the BER of the e2e SNR as

$$
\begin{aligned}
\bar{P}_{e} & =\frac{\theta_{1}^{\alpha_{1}+\beta_{1}-1} \mathcal{T}_{1} \delta}{2 \Gamma(b)(2 \pi)^{\theta_{1}-1}} \sum_{l=1}^{m} a_{l}^{b} \sum_{p=0}^{\infty} \frac{\mathcal{A}(p)}{p !} \\
& \times G_{2 \theta_{1}+2,4 \theta_{1}+1}^{3 \theta_{1}, \theta_{1}+2}\left(\frac{\mathcal{K}_{1}^{\theta_{1}}}{a_{l} \theta_{1}^{2 \theta_{1}}} \mid \begin{array}{c}
1-b, 1-p, \Delta\left(\theta_{1}, 1\right), \mathbf{\Delta}_{1} \\
\mathbf{\nabla}_{1}, \Delta\left(\theta_{1}, 0\right), 1
\end{array}\right)
\end{aligned}
$$

The asymptotic BER is given by

$$
\bar{P}_{e} \dot{\sim} \mathcal{B}(\delta, b) G_{2 \theta_{1}+1,4 \theta_{1}}^{3 \theta_{1}, \theta_{1}+1}\left(\begin{array}{c|c}
\mathcal{K}_{1}^{\theta_{1}} & \begin{array}{c}
1-b, \Delta\left(\theta_{1}, 1\right), \boldsymbol{\Delta}_{1} \\
a_{l} \theta_{1}^{2 \theta_{1}}
\end{array} \\
\mathbf{\nabla}_{1}, \Delta\left(\theta_{1}, 0\right)
\end{array}\right)
$$

with $\mathcal{B}(\delta, b) \equiv \frac{\theta_{1}^{\alpha_{1}+\beta_{1}-1} \mathcal{T}_{1} \delta}{2 \Gamma(b)(2 \pi)^{\theta_{1}-1}} \sum_{l=1}^{m} a_{l}^{b}$.

\section{E. Amount of Fading}

The $n$-th order amount of fading (AoF) is defined as [58]

$$
A o F_{\gamma}^{(n)}=\frac{\mathbb{E}\left\langle\gamma^{n}\right\rangle}{\mathbb{E}\langle\gamma\rangle^{n}}-1
$$

Therefore, we can obtain AoF for fixed-gain relay systems by substituting (19) into (46).

\section{Systems With Variable Gain Relay}

Zedini et al. [22] only considered the present system model under the fixed-gain relay scenario. In this section, we show that the multiplication theorems of Meijer [36, Eqs. 13 and 14] or [59, Eq. 79] can also be used in reducing the complexity of the CDF of the e2e SNR for CSI-assisted relay systems. While the $\mathrm{CDF}$ in this case does not enable straightforward derivation of BFHF-free performance metrics such as BER and channel capacity, we can nonetheless obtain BFHF-free expressions for OP and amount of fading (AoF).

\section{A. Statistical Properties}

1) $C D F$ of e2e SNR: In CSI-assisted relay systems, the e2e SNR is accurately approximated by [37]

$$
\gamma=\frac{\gamma_{1} \gamma_{2}}{\gamma_{1}+\gamma_{2}}
$$

The ratio (47) arises frequently in many wireless communication systems. For instance, in multiple-input-multiple-output (MIMO) AF relay systems employing orthogonal space-time block codes (OSTBCs) when no information about noise statistics is available [60], [61].

Theorem 2. The CDF of the e2e SNR for CSI-assisted relay systems is given by (48). Furthermore, the series are uniformly convergent.

$$
\begin{aligned}
F_{\gamma}(\gamma)=1 & -\frac{\theta_{1}^{\alpha_{1}+\beta_{1}-2} \mathcal{T}_{1}}{(2 \pi)^{\theta_{1}-1}} \sum_{p=0}^{\infty} \frac{\mathcal{C}(p, \gamma)}{(-1)^{p} p !} \\
& \times G_{2 \theta_{1}+1,4 \theta_{1}+1}^{4 \theta_{1}+1,0}\left(\begin{array}{c|c}
\frac{\mathcal{K}_{1}^{\theta_{1}} \gamma}{\theta_{1}^{2 \theta_{1}}} & \left.\begin{array}{c}
1-p, \Delta\left(\theta_{1}, 1\right), \mathbf{\Delta}_{1} \\
\mathbf{\nabla}_{1}, \Delta\left(\theta_{1}, 0\right), 1
\end{array}\right),
\end{array}\right.
\end{aligned}
$$

where $\mathcal{C}(0, \gamma)=1-F_{\gamma_{2}}(\gamma)$ and for $p \geq 1$,

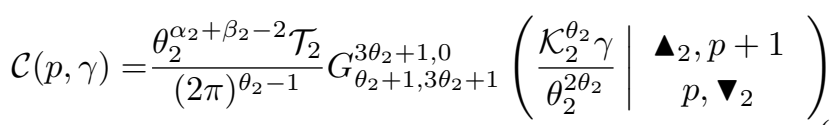

Proof. Please refer to Appendix B

Is is worth pointing out that (48) is more appealing, from a computational implementation point of view, than the seriesfree form that can be proved in a similar manner to the proof of Corollary 1 as

$$
\begin{aligned}
& F_{\gamma}(\gamma)=1-\prod_{i=1}^{2}\left(\frac{\theta_{i}^{\alpha_{i}+\beta_{i}-1} \mathcal{T}_{i}}{(2 \pi)^{\theta_{i}-1}}\right) \\
& \quad \times G_{1,0: 2 \theta_{2}+1,3 \theta_{2}+2 ; 2 \theta_{1}, 4 \theta_{1}+1}^{0,0: 3 \theta_{2}+1, \theta_{2}+1 ; 4 \theta_{1}+1,0}\left(\begin{array}{c|c}
\mathcal{K}_{2}^{\theta_{2}} \gamma / \theta_{2}^{2 \theta_{2}} & \boldsymbol{\nabla}^{\star} \\
\mathcal{K}_{1}^{\theta_{1}} \gamma / \theta_{1}^{2 \theta_{1}} & \boldsymbol{\Delta}^{\star}
\end{array}\right)
\end{aligned}
$$

where $\boldsymbol{\nabla}^{\star} \equiv 1: 0, \Delta\left(\theta_{2}, 1\right), \boldsymbol{\Delta}_{2} ; \Delta\left(\theta_{1}, 1\right), \boldsymbol{\Delta}_{1}$ and $\boldsymbol{\Delta}^{\star} \equiv$ - : $0, \boldsymbol{\nabla}_{2}, \Delta\left(\theta_{2}, 0\right), 1 ; 1, \nabla_{1}, \Delta\left(\theta_{1}, 0\right)$. Moreover, Eq. (50) is convergent as per [24, Conditions 2.61-2.64]. Another upper bound for (47) which has been used extensively in the literature due to its mathematical tractability was proposed in [62] as

$$
\gamma=\frac{\gamma_{1} \gamma_{2}}{\gamma_{1}+\gamma_{2}} \leq \min \left(\gamma_{1}, \gamma_{2}\right)
$$

The approximate CDF of $\gamma$ based on (51) can be shown to be

$$
\begin{aligned}
F_{\gamma}(\gamma) & =1-\tilde{F}_{\gamma_{1}}(\gamma) \tilde{F}_{\gamma_{2}}(\gamma) \\
& =\prod_{i=1}^{2} \theta_{i} \mathcal{T}_{i} G_{2,4}^{4,0}\left(\begin{array}{l|c}
\mathcal{K}_{i} \gamma^{\frac{1}{\theta_{i}}} & 1, \xi_{i}^{2}+1 \\
\xi_{i}^{2}, \alpha_{i}, \beta_{i}, 0
\end{array}\right)
\end{aligned}
$$

which corresponds to the asymptotic and leading term (i.e. term of series at $p=0$ ) in (48). Therefore, it is expected that $\min \left(\gamma_{1}, \gamma_{2}\right)$ is a good approximate only at high SNR regimes as shown on Figure 10.

2) Exact and asymptotic general moments of e2e SNR: The generalized moments may be estimated by substituting (48) into $\mathbb{E}\left\langle\gamma^{n}\right\rangle=n \int_{0}^{\infty} \gamma^{n-1}\left[1-F_{\gamma}(\gamma)\right] d \gamma$ and applying [42, Eq. 2.24.1(1)] so that we obtain, after some simplifications, the compact form

$$
\begin{aligned}
\mathbb{E}\left\langle\gamma^{n}\right\rangle= & n \prod_{i=1}^{2}\left(\frac{\theta_{i}^{\alpha_{i}+\beta_{i}-1} \mathcal{T}_{i}}{(2 \pi)^{\theta_{i}-1}}\right)\left(\frac{\theta_{1}^{2 \theta_{1}}}{\mathcal{K}_{1}^{\theta_{1}}}\right)^{n} \sum_{p=0}^{\infty} \frac{(-1)^{p}}{p !} \\
& \times G_{4 \theta_{1}+\theta_{2}+2,2 \theta_{1}+3 \theta_{2}+2}^{3 \theta_{2}+1,4 \theta_{1}+1}\left(\frac{\theta_{1}^{2 \theta_{1}} \mathcal{K}_{2}^{\theta_{2}}}{\theta_{2}^{2 \theta_{2}} \mathcal{K}_{1}^{\theta_{1}}} \begin{array}{c}
\mathbf{Q}(n, p) \\
\mathbf{Q}(n, p)
\end{array}\right)
\end{aligned}
$$

where $\theta_{1}^{2 \theta_{1}} \mathcal{K}_{2}^{\theta_{2}} \neq \theta_{2}^{2 \theta_{2}} \mathcal{K}_{1}^{\theta_{1}}$ with $\boldsymbol{\Lambda}$ and given respectively by $\boldsymbol{\mathbf { A }}(n, p) \equiv-n, 1-n-\boldsymbol{\nabla}_{1}^{\prime}, \mathbf{\Delta}_{2}, p+1$ and $\boldsymbol{\hat { s }}(n, p) \equiv p, \mathbf{\nabla}_{2}, p-$ $n, 1-n-\boldsymbol{\Delta}_{1}^{\prime}$. Using the method from the proof of Corollary 1, (53) can be simplified to

$$
\begin{aligned}
& \mathbb{E}\left\langle\gamma^{n}\right\rangle=\frac{\theta_{1}^{2 n \theta_{1}}}{\Gamma(n) \mathcal{K}_{1}^{n \theta_{1}}} \prod_{i=1}^{2}\left(\frac{\theta_{i}^{\alpha_{i}+\beta_{i}-1} \mathcal{T}_{i}}{(2 \pi)^{\theta_{i}-1}}\right) \\
& \times G_{4 \theta_{1}+\theta_{2}+1,2 \theta_{1}+3 \theta_{2}+1}^{3 \theta_{2}+1,4 \theta_{1}+1}\left(\begin{array}{c|c}
\frac{\theta_{1}^{2 \theta_{1}} \mathcal{K}_{2}^{\theta_{2}}}{\theta_{2}^{2 \theta_{2}} \mathcal{K}_{1}^{\theta_{1}}} & -n, 1-n-\boldsymbol{\nabla}_{1}^{\prime}, \mathbf{\Delta}_{2} \\
& 0, \mathbf{\nabla}_{2}, 1-n-\mathbf{\Delta}_{1}^{\prime}
\end{array}\right)
\end{aligned}
$$


with $\theta_{1}^{2 \theta_{1}} \mathcal{K}_{2}^{\theta_{2}} \neq \theta_{2}^{2 \theta_{2}} \mathcal{K}_{1}^{\theta_{1}}$. Finally, the asymptotic $n$-th moment of $\gamma$ can be deduced by setting $p=0$ in (53) yielding

$$
\begin{aligned}
& \mathbb{E}\left\langle\gamma^{n}\right\rangle \dot{\sim} \frac{n \theta_{1}^{2 n \theta_{1}}}{\mathcal{K}_{1}^{n \theta_{1}}} \prod_{i=1}^{2}\left(\frac{\theta_{i}^{\alpha_{i}+\beta_{i}-1} \mathcal{T}_{i}}{(2 \pi)^{\theta_{i}-1}}\right) \\
& \times G_{4 \theta_{1}+\theta_{2}+1,2 \theta_{1}+3 \theta_{2}+1}^{3 \theta_{2}+1,4 \theta_{1}}\left(\begin{array}{l|l}
\frac{\theta_{1}^{2 \theta_{1}} \mathcal{K}_{2}^{\theta_{2}}}{\theta_{2}^{2 \theta_{2}} \mathcal{K}_{1}^{\theta_{1}}} & \left.\begin{array}{l}
1-n-\boldsymbol{\nabla}_{1}^{\prime}, \boldsymbol{\Delta}_{2}, 1 \\
0,1-n-\mathbf{\Delta}_{1}^{\prime}
\end{array}\right)
\end{array}\right)
\end{aligned}
$$

where $\theta_{1}^{2 \theta_{1}} \mathcal{K}_{2}^{\theta_{2}} \neq \theta_{2}^{2 \theta_{2}} \mathcal{K}_{1}^{\theta_{1}}$. To the best of our knowledge, the expression in (54) has not been reported before in the open literature.

Remark 2. It worth mentioning that the model in Eq. (47) can be extended to a triple-hop system as

$$
\gamma=\frac{\gamma_{1} \gamma_{2} \gamma_{3}}{\gamma_{1} \gamma_{2}+\gamma_{1} \gamma_{3}+\gamma_{2} \gamma_{3}}=\frac{\gamma_{3}\left(\frac{\gamma_{1} \gamma_{2}}{\gamma_{1}+\gamma_{2}}\right)}{\gamma_{3}+\left(\frac{\gamma_{1} \gamma_{2}}{\gamma_{1}+\gamma_{2}}\right)}
$$

Therefore, we can use a method similar to that used in the proof of Theorem 2 to obtain the CDF of $\gamma$ as a single series of a product of a univariate Meijer G-function and a BMGF. The series-free form can be expressed as a trivariate Meijer G-function.

\section{NUMERICAL EXPERIMENTS AND SIMULATIONS}

In this section, we investigate and verify the validity of the analytical formulae derived in the preceding sections through Monte Carlo simulations. For all curves employing Monte Carlo simulations, $10^{7}$ random $\Gamma \Gamma$ variables are generated using the MATLAB ${ }^{\circledR}$ code from [45]. (Note that the meijer G-function of each PDF must be written in the form $G_{n, m}^{m, 0}(\cdot)$ or the code will not work.) All series have been truncated at $P=25$. Following in line with objectives of the paper outlined in the introduction, we will avoid repeating the results already presented in [22] to avoid redundancy. More attention will be given to studying the results of Sections IV-A and V. Except for Figures 5, 6 and 8, the simulations have been setup in a similar manner to [3] with turbulence severity varied as follows : The combination $\alpha=2.902$ and $\beta=2.51$ represents weak turbulence, $\alpha=2.296$ and $\beta=1.822$ denotes moderate turbulence and $\alpha=2.094$ and $\beta=1.342$ stands for strong turbulence conditions. Pointing error level is set as $\xi=1.1$ for severe pointing errors and $\xi=6.7$ denotes low pointing errors. For the fixed-gain relay system, the gain has been fixed at $\mathcal{G}=1$ throughout the simulations. Without any loss of generality, we also assume that $\gamma_{1}$ and $\gamma_{2}$ are independent and identically distributed $\Gamma \Gamma$ random variables. As expected, in Figure 5, we observe that ergodic capacity improves substantially with reduction in turbulence or pointing error severity. It is also worth noting that the asymptotic ergodic capacity based on (26), which is essentially the ergodic capacity in the $\mathrm{S}-\mathrm{R}$ link, is a very good approximate at high SNR regimes, i.e. for $\bar{\gamma} \geq 15 \mathrm{~dB}$. This is a far simpler form than dealing with the asymptotic expansion of Eq. (15) as was the case in [22]. Furthermore, the asymptotic approximates are well behaved even at low SNR regimes since they do not blowup. Since the ergodic capacity for IM/DD is a lower bound, we can only conclude that HD outperforms IM/DD but we cannot quantify the difference. Figure 6 presents the outage probability based on Eq. (8). It is evident that the system performance improves with improvement in pointing error and turbulence conditions. All these outcomes are in agreement with existing results reported in the literature and also corroborate the correctness of our proposed results. It is clear from Figures 5, 6 and 8 that a dual-hop system setup outperforms a single link system and this improves with increase in average electrical SNR. In Figure 7, we investigate the capacity of the system under different adaptive transmission schemes using HD. It can be deduced that channel capacity under OPRA scheme performs better than rest of the schemes, especially at $0 \mathrm{~dB} \leq \bar{\gamma} \leq 15 \mathrm{~dB}$, and this is in line with the definition of OPRA capacity as the highest achievable capacity. This is further supported by Figure (8). However, the ORA and OPRA capacities are almost indistinguishable at high SNR regimes. Since implementation of the OPRA scheme is much more complex that the ORA policy, it is recommended to employ OPRA scheme only at low SNR regimes to maximize capacity. As expected, the TIFR capacity approaches the ORA capacity from below as $\gamma_{0}$ decreases. In the case of effective capacity, Figure 9 suggests that as the product between $\phi, B$ and $\boldsymbol{T}_{f}$ (QoS exponent, system bandwidth and fading block/frame length) decreases, the effective capacity increases to match the ergodic capacity. In Figure 10, we compare the accuracy of $\min \left(\gamma_{1}, \gamma_{2}\right)$ against $\gamma_{1} \gamma_{2} /\left(\gamma_{1}+\gamma_{2}\right)$ and it is clearly evident that the former is only good at high SNR regimes.

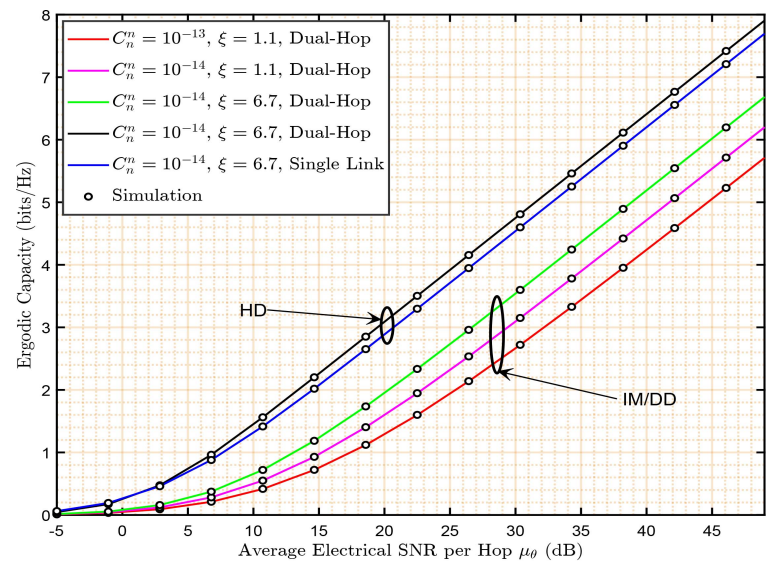

Figure 5: Ergodic Capacity under different detection schemes, atmospheric turbulence and pointing error severity with $\lambda=$ $1550 \mathrm{~nm}$ over a propagation distance of $2 \mathrm{~km}$.

\section{CONCLUSIONS}

In this paper, we have revisited the performance analysis of an all-optical dual-hop FSO transmission system and presented a new approach to simplify the mathematical intractability in the derivation of the CDF and PDF of the e2e SNR. The proposed analytical formulae enable easy implementation in latest versions of standard computing systems such as MATLAB ${ }^{\circledR}$, MATHEMATICA $^{\circledR}$ and MAPLE ${ }^{\circledR}$. In particular, the derived CDF expressions make it considerably easier to study channel capacity under different adaptive transmission 


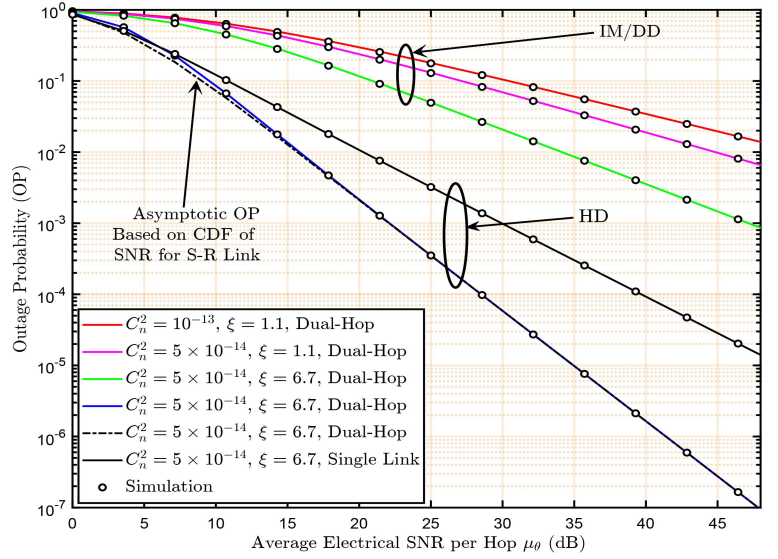

Figure 6: Outage probability for Dual-Hop and Single Link under different atmospheric turbulence and pointing error severity for a propagation distance of $2 \mathrm{~km}$ and $\lambda=1050 \mathrm{~nm}$.

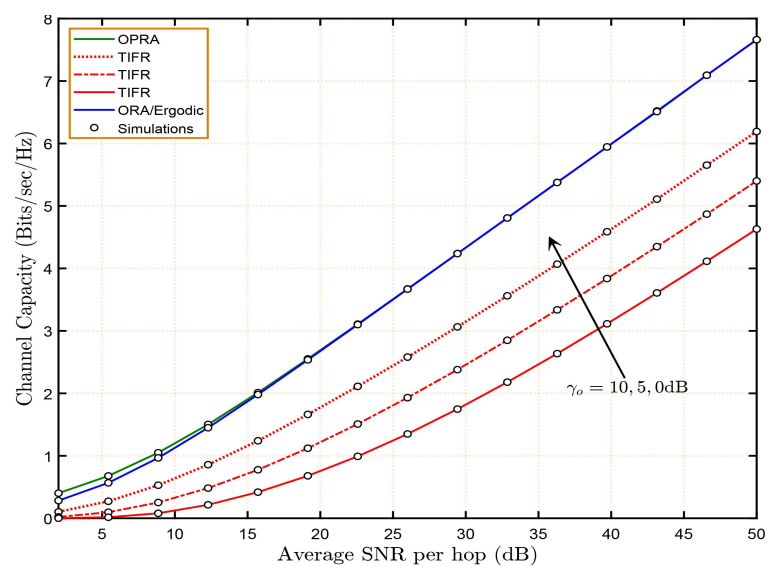

Figure 7: Channel capacity under strong atmospheric turbulence and high pointing errors using different adaptive transmission schemes.

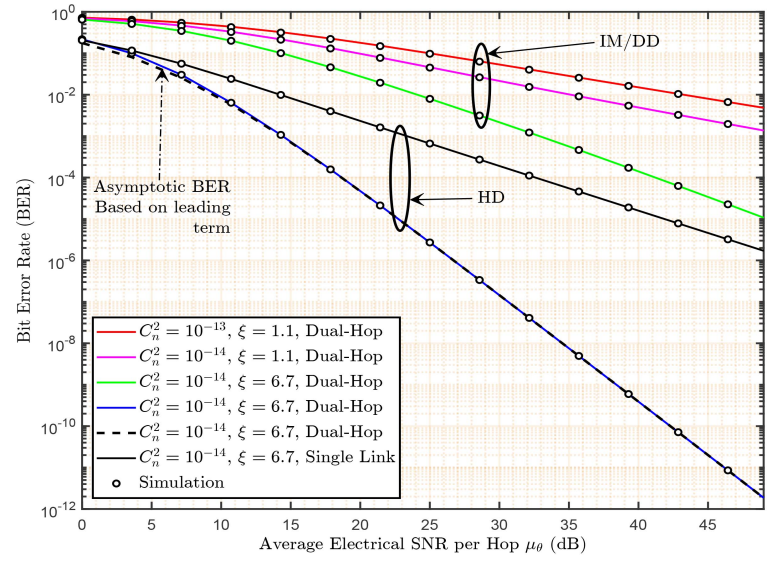

Figure 8: Bit Error Rate at different turbulence and pointing error severity with $\lambda=1550 \mathrm{~nm}$ and a propagation distance of $2 \mathrm{~km}$.

schemes for AF-based dual-hop FSO systems, which is a problem that has not been addressed before in the literature for the present system model. While the evaluation of some of

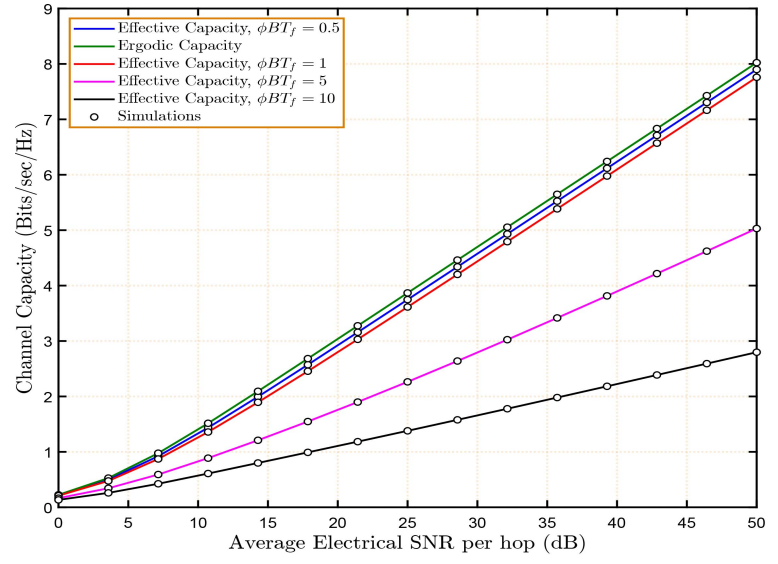

Figure 9: Ergodic Capacity Vs Effective channel capacity under weak atmospheric turbulence and low pointing errors showing the effect of variation in the QoS and/or fading block/frame length.

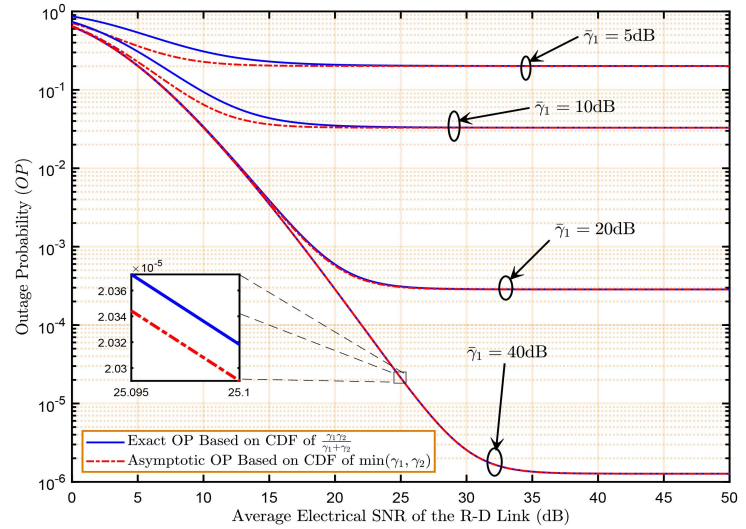

Figure 10: CSI-assisted relay systems's outage probability based on (48) under weak atmospheric turbulence and low pointing errors, showing the effect of increase in average SNR of the R-D link while that of the S-R link is kept constant.

the Meijer G-functions in the present work, particularly under IM/DD, maybe be slow, the derived expressions can still be useful for approximate and asymptotic analyses for very low truncations.

\section{APPENDIX A}

DERIVATION OF CDF AND PDF OF $\gamma$ FOR FIXED-GAIN RELAYING AND PROOF OF ITS UNIFORM CONVERGENCE

\section{A. Mathematical Derivation}

Proof: From Bayes theory, we have that the CDF of $\gamma$ can be written as

$$
F_{\gamma}(\gamma)=\int_{0}^{\infty} \operatorname{Pr}\left(\frac{\gamma_{1} \gamma_{2}}{\gamma_{2}+\mathcal{G}}<\gamma \mid \gamma_{2}\right) f_{2}\left(\gamma_{2}\right) d \gamma_{2}
$$


Now, using (2) and (6) in (57), we have that

$$
\begin{aligned}
F_{\gamma}(\gamma)= & \frac{\mathcal{T}_{1} \mathcal{T}_{2} \theta_{1}^{\alpha_{1}+\beta_{1}-2}}{(2 \pi)^{\theta_{1}-1}} \int_{0}^{\infty} \frac{1}{x} G_{1,3}^{3,0}\left(\mathcal{K}_{2} x^{\frac{1}{\theta_{2}}} \mid \begin{array}{c}
\xi_{2}^{2}+1 \\
\xi_{2}^{2}, \alpha_{2}, \beta_{2}
\end{array}\right) \\
& \times \underbrace{G_{2 \theta_{1}, 4 \theta_{1}}^{3 \theta_{1}, \theta_{1}}\left(\frac{\mathcal{K}_{1}^{\theta_{1}} \gamma}{\theta_{1}^{2 \theta_{1}}}\left(1+\frac{\mathcal{G}}{x}\right) \mid \begin{array}{c}
\Delta\left(\theta_{1}, 1\right), \mathbf{\Delta}_{1} \\
\mathbf{\nabla}_{1}, \Delta\left(\theta_{1}, 0\right)
\end{array}\right)}_{\mathcal{H}} d x
\end{aligned}
$$

Observing that $1+\frac{\mathcal{G}}{x}>\frac{1}{2}$ for all $x \in \mathbb{R}^{+}$within the integral and employing [36, Eq. 13], $\mathcal{H}$ can be expanded as

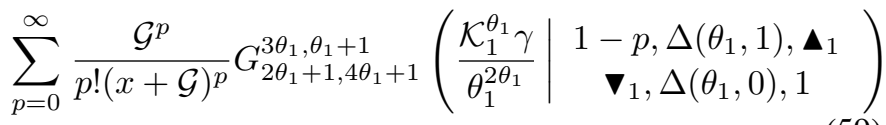

Therefore, substituting (59) into (58), the CDF of $\gamma$ can be derived using [42, Eq. 2.24.2(4)] as given in (8). Note that an equivalent $\mathrm{CDF}$ expression can be obtained by factorizing $\mathcal{H}$ using [59, Eq. 79] with $l=0$. The PDF of $\gamma$ may be obtained by deriving (8) once with respect to $\gamma$ using [42, Eq. 8.2.2(40)]. On the other hand, if one considered using the CDF of $\gamma_{1}$ as given in the second form of (7), then it is fairly straightforward to show that [63, Eq. 18] and [36, Eq. 14] lead to the CDF and PDF of the e2e SNR being given in (11) and (12), respectively. For the third and final CDF, we use the first form of (6) and apply [36, Eq. 13] to extract the term $\left\{1-\gamma_{2}^{\frac{1}{\theta_{1}}} /\left[\left(\gamma_{2}+\mathcal{G}\right)^{\frac{1}{\theta_{1}}}\right]\right\}^{p}$ and then use the binomial expansion to obtain the CDF of $\gamma$ as given in (13).

Remark 3. The shifted term $(x+\mathcal{G}) / x$ is what makes the evaluation of the integral (58) a daunting task. As observed from (59), the novelty of the proposed method of deriving CDFs is based on the observation that either of [36, Eqs. 13 and 14], [43, Theorem 2, Eq. 33] or [59, Eq. 79] can be used in extracting this term out of the Meijer G-function, which greatly simplifies the integration.

\section{B. Proof of Uniform Convergence}

In order to prove the uniform convergence of the CDFs and PDFs given in Table I, we first start by recalling Abel's criterion for test of uniform convergence in $\mathbb{R}[64$, Theorem $0.304]$ :

Theorem 3 (Abel's Test For Uniform Convergence in $\mathbb{R}$ ). Suppose that the series $\sum_{p=1}^{\infty} f_{p}(x)$ converges uniformly in a region $R$. Furthermore, suppose that the set of functions $g_{p}(x)$ constitutes (for each $x$ ) a monotonic sequence, and that these functions are uniformly bounded; i.e. suppose that there exists a number $L$ such that the inequalities

1) $\left|g_{p}(x)\right| \leq L$ hold for all $p$ and $x$. Then, the series

2) $\sum_{p=1}^{\infty} f_{p}(x) g_{p}(x)$ converges uniformly in the region $R$.

Now, considering CDF form (8), define the functions $f_{p}(x)$ and $g_{p}(x)$ respectively as

$$
\begin{aligned}
& f_{p}(x)=\frac{\theta_{1}^{\alpha_{1}+\beta_{1}-2} \mathcal{T}_{1}}{(2 \pi)^{\theta_{1}-1}} \frac{1}{p !} \\
& \times G_{2 \theta_{1}+1,4 \theta_{1}+1}^{3 \theta_{1}, \theta_{1}+1}\left(\begin{array}{c|c}
\mathcal{K}_{1}^{\theta_{1}} x & 1-p, \Delta\left(\theta_{1}, 1\right), \mathbf{\Delta}_{1} \\
\hline \theta_{1}^{2 \theta_{1}} & \mathbf{\nabla}_{1}, \Delta\left(\theta_{1}, 0\right), 1
\end{array}\right)
\end{aligned}
$$

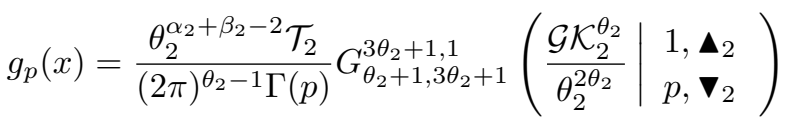

for $p \geq 1$ with $g_{0}(x)=1$. Next, we invoke [42, Eq. 6.11.1(5)] to obtain

$$
\begin{aligned}
& \sum_{p=0}^{\infty} \frac{1}{p !} G_{2 \theta_{1}+1,4 \theta_{1}+1}^{3 \theta_{1}, \theta_{1}+1}\left(\frac{\mathcal{K}_{1}^{\theta_{1}} x}{\theta_{1}^{2 \theta_{1}}} \mid \begin{array}{c}
1-p, \Delta\left(\theta_{1}, 1\right), \mathbf{\Delta}_{1} \\
\mathbf{\nabla}_{1}, \Delta\left(\theta_{1}, 0\right), 1
\end{array}\right) \\
& =\frac{\Gamma\left(\frac{\xi_{1}^{2}}{2}\right) \Gamma\left(\frac{\alpha_{1}}{2}\right) \Gamma\left(\frac{\alpha_{1}+1}{2}\right) \Gamma\left(\frac{\beta_{1}}{2}\right)}{\left[\Gamma\left(\frac{\beta_{1}+1}{2}\right)\right]^{-1} \Gamma\left(\frac{\xi_{1}^{2}+2}{2}\right)}=\frac{(2 \pi) \Gamma\left(\alpha_{1}\right) \Gamma\left(\beta_{1}\right) \Gamma\left(\xi_{1}^{2}\right)}{2^{\alpha_{1}+\beta_{1}-2} \Gamma\left(\xi_{1}^{2}+1\right)}
\end{aligned}
$$

for $\theta_{1}=2$ and

$$
\sum_{p=0}^{\infty} \frac{1}{p !} G_{2,4}^{3,1}\left(\mathcal{K}_{1} x \mid \begin{array}{c}
1-p, \xi_{1}^{2}+1 \\
\xi_{1}^{2}, \alpha_{1}, \beta_{1}, 0
\end{array}\right)=\frac{\Gamma\left(\alpha_{1}\right) \Gamma\left(\beta_{1}\right) \Gamma\left(\xi_{1}^{2}\right)}{\Gamma\left(\xi_{1}^{2}+1\right)}
$$

for $\theta_{1}=1$. That is, $\sum_{p=0}^{\infty} f_{p}(x)$ converges pointwise to 1 . In order to establish the uniform convergence of $\sum_{p=0}^{\infty} f_{p}(x)$ on $\mathbb{R}^{+}$, we need to show that the sequence of partial sums $\left\{S_{k}(x)\right\}_{k \in \mathbb{N}_{0}}$, where $S_{k}(x)=\sum_{p=0}^{k} f_{p}(x)$, converges uniformly to the pointwise limit 1 as $k \rightarrow \infty$. Since $\mathbb{R}$ is a complete metric space, the uniform convergence of $\left\{S_{k}(x)\right\}_{k \in \mathbb{N}_{0}}$ on $\mathbb{R}^{+}$can be characterized as a simple convergence with respect to the supremum metric $d_{k}=\sup _{x \in \mathbb{R}^{+}}\left|S_{k}(x)-1\right|$. Thanks to the flexibility of the proposed $\stackrel{x \in \mathbb{R}^{+}}{\mathrm{CDF}}$ forms, an explicit expression for $S_{k}(x)$ can be obtained using the simple method from the proof of Corollary 1 as

$$
\begin{aligned}
S_{k}(x)= & \frac{\theta_{1}^{\alpha_{1}+\beta_{1}-2} \mathcal{T}_{1}}{(2 \pi)^{\theta_{1}-1}} \frac{1}{k !} \\
& \times G_{2 \theta_{1}+1,4 \theta_{1}+1}^{3 \theta_{1}, \theta_{1}+1}
\end{aligned}\left(\begin{array}{c|c}
\frac{\mathcal{K}_{1}^{\theta_{1}} x}{\theta_{1}^{2 \theta_{1}}} & \left.\begin{array}{c}
-k, \Delta\left(\theta_{1}, 1\right), \mathbf{\Delta}_{1} \\
\mathbf{\nabla}_{1}, \Delta\left(\theta_{1}, 0\right), 0
\end{array}\right) .
\end{array}\right.
$$

Now, using [65, Eq. 5.11.12], we have that $\frac{\Gamma(1+k+s)}{\Gamma(1+k)} \sim k^{s}$ as $k \rightarrow \infty$ resulting in

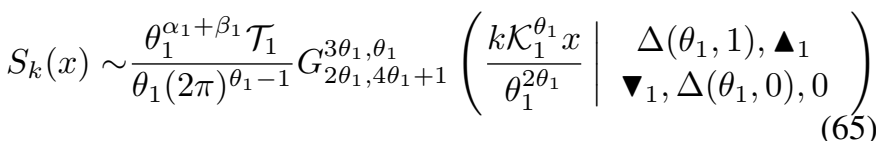

for large $k$. Comparing (65) with the CDF of $\gamma_{1}$ (i.e. the first equation of (6)), we note that the two expressions are identical save the extra factor $1 / \Gamma(1+s)$ in the integrand of the Meijer G-function in (65) whose effect is negligible for large $k$. It follows from properties of CDFs that $F_{\gamma_{1}}(z) \rightarrow 1$ as $z \rightarrow \infty$ and the latter implies that $S_{k}(x) \rightarrow 1$ as $k \rightarrow \infty$. This can also be observed from Figure 12. Therefore, $d_{k} \rightarrow 0$ as $k \rightarrow \infty$ and thus the uniform convergence of $\sum_{p=0}^{\infty} f_{p}(x)$ is established. It is also clear that for each $x \in \mathbb{R}^{+},\left|g_{p}(x)\right| \leq 1$ and $g_{p+1}(x) \leq$ $g_{p}(x)$ for all $p$ due to the presence of the factor $1 / \Gamma(p)$. The latter implies that $\left\{g_{p}(x)\right\}_{p \in \mathbb{N}_{0}}$ is a monotonic decreasing sequence as depicted in Figure 13. Finally, in order to ensure that the exchange of differentiation and summation are permissible, i.e. $\frac{d}{d x} \sum_{p=0}^{\infty} f_{p}(x) g_{p}(x)=\sum_{p=0}^{\infty} \frac{d}{d x}\left[f_{p}(x) g_{p}(x)\right]$, we need to prove that $\sum_{p=0}^{\infty} \frac{d}{d x}\left[f_{p}(x) g_{p}(x)\right]$ also converges uniformly on $\mathbb{R}^{+}$. Therefore, following the same procedure as above, we can show that $\sum_{p=0}^{\infty} \frac{d}{d x}\left[f_{p}(x) g_{p}(x)\right]$ and its sequence of partial sums converge point-wise and uniformly to 
0 , respectively. This completes the proof and justifies the termwise differentiation and integration as well as the interchange of integral and summation signs performed throughout the paper. The proofs for the rest of the CDF and PDF forms in Table I can be derived in a similar manner.

\section{Upper Bound for the Truncation Error}

Since, [43, Theorem 2] is based on a Taylor series expansion, we use to the Cauchy form of the remainder [64, Eq. $0.317(4)]$ for truncating the series representation at $n$ terms which can be derived as

$$
\begin{aligned}
& \mathcal{R}_{n}(x)=\frac{\varrho^{n}}{n !}\left(\frac{\mathcal{G}}{x+\varrho \mathcal{G}}\right)^{n+1} \\
& \times G_{2 \theta_{1}+1,4 \theta_{1}+1}^{3 \theta_{1}, \theta_{1}+1}\left(\frac{\mathcal{K}_{1}^{\theta_{1}} \gamma(x+\mathcal{G})}{\theta_{1}^{2 \theta_{1}}(x+\varrho \mathcal{G})} \mid \begin{array}{c}
-n, \Delta\left(\theta_{1}, 1\right), \mathbf{\Delta}_{1} \\
\mathbf{\nabla}_{1}, \Delta\left(\theta_{1}, 0\right), 1
\end{array}\right)
\end{aligned}
$$

where $0<\varrho<1$. As $n \rightarrow \infty, \frac{\Gamma(1+n+s)}{\Gamma(1+n)} \sim n^{s}[65$, Eq. 5.11.12] so that

$$
\begin{aligned}
& \mathcal{R}_{n}(x) \sim \frac{1}{\varrho}\left(\frac{\varrho \mathcal{G}}{x+\varrho \mathcal{G}}\right)^{n+1} \\
& \times G_{2 \theta_{1}, 4 \theta_{1}+1}^{3 \theta_{1}, \theta_{1}}\left(\frac{n \mathcal{K}_{1}^{\theta_{1}} \gamma(x+\mathcal{G})}{\theta_{1}^{2 \theta_{1}}(x+\varrho \mathcal{G})} \mid \begin{array}{c}
\Delta\left(\theta_{1}, 1\right), \mathbf{\Lambda}_{1} \\
\mathbf{v}_{1}, \Delta\left(\theta_{1}, 0\right), 1
\end{array}\right) \rightarrow 0
\end{aligned}
$$

It is not easy to determine the sign of the Meijer G-function in (66) as $x$ or $n$ varies. However, from Figure 11, we note that $\left|\mathcal{R}_{n}(x)\right|$ exhibits a similar monotonic decreasing property which was observed for (61) in Figure 13. Therefore, assuming the latter, $\left|\mathcal{R}_{n}(x)\right|$ is upper-bounded by $\mathcal{R}_{u}(n)=$ $\sup \left\{\left|\mathcal{R}_{n}(x)\right|: x \in \mathbb{R}^{+}\right\}$, where

$\mathcal{R}_{u}(n)=\frac{1}{n ! \varrho}\left|G_{2 \theta_{1}+1,4 \theta_{1}+1}^{3 \theta_{1}, \theta_{1}+1}\left(\frac{\mathcal{K}_{1}^{\theta_{1}} \gamma}{\theta_{1}^{2 \theta_{1}} \varrho} \mid \begin{array}{c}-n, \Delta\left(\theta_{1}, 1\right), \mathbf{\Delta}_{1} \\ \mathbf{\nabla}_{1}, \Delta\left(\theta_{1}, 0\right), 1\end{array}\right)\right|$.

An upper-bound for the error of truncating the CDF (8) after $n$ terms can thus be obtained by substituting (68) into (58) which yields $\mathcal{R}_{u}(n)$ as well. Therefore, to get within $\epsilon$ of the exact CDF of $\gamma$, we would have to determine a $n$ such that $\mathcal{R}_{u}<\epsilon$. While this is clearly not rigorous enough, it should suffice to give us a general idea on the convergence rates of the seriesbased formulae proposed in the present work. Finally, for the variable gain scenario, a similar procedure can be followed to obtain the remainder as

$$
\begin{aligned}
\mathcal{R}_{n}(x) & =\frac{(-1)^{n+1} \varrho^{n}}{n !}\left(\frac{\gamma}{x+\varrho \gamma}\right)^{n+1} \\
& \times G_{2 \theta_{1}+1,4 \theta_{1}+1}^{4 \theta_{1}+1,0}\left(\frac{\mathcal{K}_{1}^{\theta_{1}} \gamma(x+\gamma)}{\theta_{1}^{2 \theta_{1}}(x+\varrho \gamma)} \mid \begin{array}{c}
-n, \Delta\left(\theta_{1}, 1\right), \mathbf{\Delta}_{1} \\
1, \mathbf{\nabla}_{1}, \Delta\left(\theta_{1}, 0\right)
\end{array}\right)
\end{aligned}
$$

\section{APPENDIX B}

\section{DERIVATION OF THE CDF AND PDF OF $\gamma$ UNDER}

\section{CSI-ASSISTED RELAYING}

6]

Proof: The CDF of $\gamma$ can easily be shown to be [66, Eq.

$$
F_{\gamma}(\gamma)=1-\int_{0}^{\infty} \tilde{F}_{\gamma_{1}}\left[\gamma\left(1+\frac{\gamma}{x}\right)\right] f_{\gamma_{2}}(x+\gamma) d x
$$

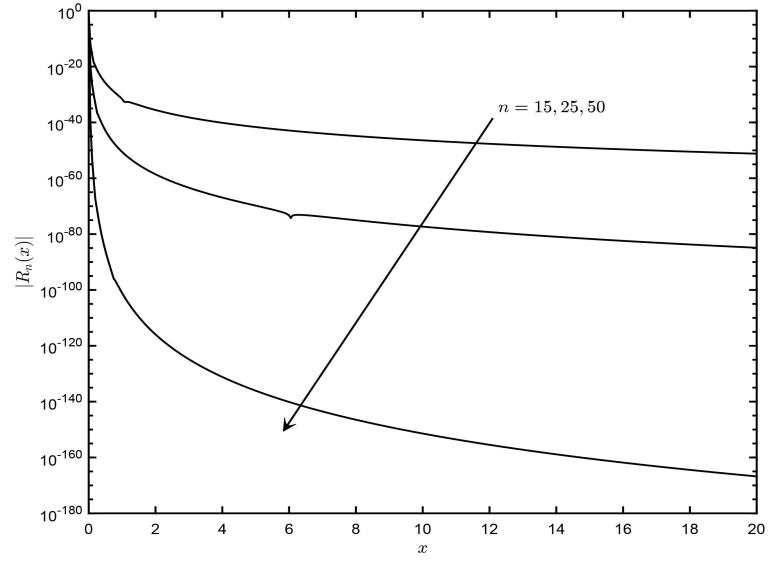

Figure 11: The truncation error $\mathcal{R}_{n}(x)$ showing the effect of increase in $n$ for moderate turbulence and strong pointing errors with $\varrho=0.01$.

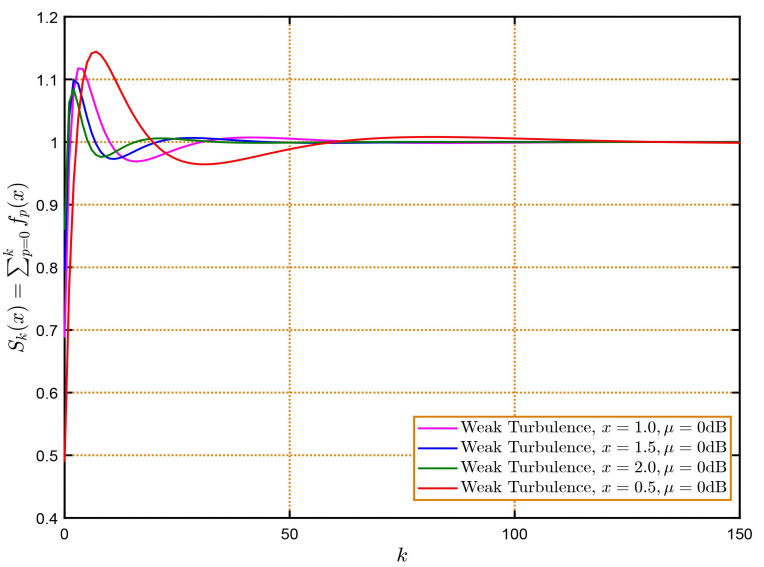

Figure 12: Several curves showing the convergence of $S_{k}(x)$ as well as the effect of the increase in the involved Meijer G-function argument using heterodyne detection.

Substituting (7) and (2) into (70) and applying [36, Eq. 14] yields a BFHF-free approximate CDF of $\gamma$ as given in (48), where $\mathcal{C}(p, \gamma)$ is given by

$$
\frac{\theta_{2}^{\alpha_{2}+\beta_{2}-2} \mathcal{T}_{2}}{(2 \pi)^{\theta_{2}-1}} \int_{0}^{\infty} \frac{\gamma^{p} d x}{(x+\gamma)^{p+1}} G_{\theta_{2}, 3 \theta_{2}}^{3 \theta_{2}, 0}\left(\frac{\mathcal{K}_{2}^{\theta_{2}}}{\theta_{2}^{2 \theta_{2}}}(x+\gamma) \mid \begin{array}{c}
\mathbf{\Delta}_{2} \\
\mathbf{\nabla}_{2}
\end{array}\right)
$$

The integral $\mathcal{C}(p, \gamma)$ can be evaluated by setting $x=z-\gamma$ and then invoking [42, Eq. 2.24.2(3)] so that we obtain (49). The proof of uniform convergence of the series follows along the lines of the proof given for Theorem 1 in Appendix A.

\section{REFERENCES}

[1] S. Hranilovic, "Trends and progress in optical wireless communications," in 2017 Optical Fiber Communications Conference and Exhibition (OFC). IEEE, 2017, pp. 1-3.

[2] A. A. Farid and S. Hranilovic, "Outage capacity optimization for free-space optical links with pointing errors," IEEE/OSA Journal of Lightwave Technology, vol. 25, no. 7, pp. 1702-1710, 2007.

[3] E. Zedini, I. S. Ansari, and M.-S. Alouini, "Performance analysis of mixed Nakagami- $m$ and gamma-gamma dual-hop FSO transmission systems," IEEE Photonics Journal, vol. 7, no. 1, pp. 1-20, 2015. 


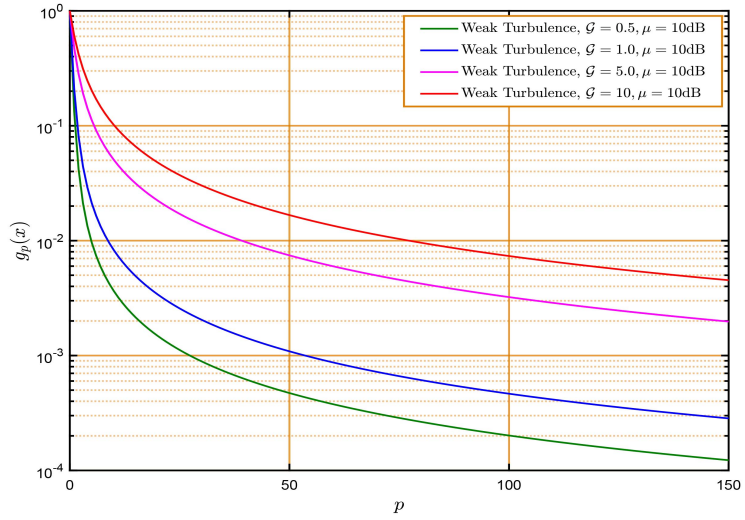

Figure 13: Depiction of the decreasing monotonicity of $\left\{g_{p}(x)\right\}_{p \in \mathbb{N}_{0}}$ as well as the effect of the increase in the involved Meijer G-function argument using heterodyne detection.

[4] M. Safari and M. Uysal, "Relay-assisted free-space optical communication," IEEE Transactions on Wireless Communications, vol. 7, no. 12 pp. 5441-5449, 2008.

[5] H. Al-Quwaiee, I. Ansari, and M.-S. Alouini, "On the maximum and minimum of double generalized gamma variates with applicaitons to the performance of free-space optical communication systems," IEEE Transactions on Vehicular Technology, vol. 65, no. 11, pp. 8822-8831, 2016.

[6] L. C. Andrews, R. L. Phillips, and C. Y. Hopen, Laser beam scintillation with applications. SPIE press, 2001, vol. 99.

[7] A. A. Farid and S. Hranilovic, "Outage capacity for MISO intensitymodulated free-space optical links with misalignment," IEEE/OSA Journal of Optical Communications and Networking, vol. 3, no. 10, pp. 780789, 2011.

[8] I. S. Ansari, F. Yilmaz, and M.-S. Alouini, "Impact of pointing errors on the performance of mixed RF/FSO dual-hop transmission systems," IEEE Wireless Communications Letters, vol. 2, no. 3, pp. 351-354, 2013.

[9] E. Zedini, H. M. Oubei, A. Kammoun, M. Hamdi, B. S. Ooi, and M.-S. Alouini, "A new simple model for underwater wireless optical channels in the presence of air bubbles," in GLOBECOM 2017-2017 IEEE Global Communications Conference. IEEE, 2017, pp. 1-6.

[10] R. Barrios and F. Dios, "Exponentiated weibull model for the irradiance probability density function of a lazer beam propagating through atmospheric turbulence," Optica \& Laser Technology, vol. 45, pp. 13-20, 2013.

[11] A. Jurado-Navas, J. M. Garrido-Balsells, J. F. Paris, and A. PuertaNotario, "A unifying statistical model for atmospheric optical scintillation," arXiv preprint arXiv:1102.1915, 2011.

[12] G. S. Rabelo, U. S. Dias, and M. D. Yacoub, "The $\kappa-\mu$ extreme distribution: Characterizing severe fading conditions," in Microwave and Optoelectronics Conference (IMOC), 2009 SBMO/IEEE MTT-S International. IEEE, 2009, pp. 244-248.

[13] M. D. Yacoub, "The $\alpha-\mu$ distribution: A physical fading model for the stacy distribution," IEEE Transactions on Vehicular Technology, vol. 56, no. 1, pp. 27-34, 2007.

[14] G. Fraidenraich and M. D. Yacoub, "The $\alpha-\eta-\mu$ and $\alpha-\kappa-\mu$ fading distributions," in Spread Spectrum Techniques and Applications, 2006 IEEE Ninth International Symposium on. IEEE, 2006, pp. 16-20.

[15] E. Jakeman and P. Pusey, "Significance of K distributions in scattering experiments," Physical Review Letters, vol. 40, no. 9, p. 546, 1978.

[16] R. L. Phillips and L. C. Andrews, "Measured statistics of laser-light scattering in atmospheric turbulence," JOSA, vol. 71, no. 12, pp. 14401445,1981

[17] G. Parry, "Measurement of atmospheric turbulence induced intensity fluctuations in a laser beam," Optica Acta: International Journal of Optics, vol. 28, no. 5, pp. 715-728, 1981.

[18] G. N. Kamga, S. Aïssa, T. R. Rasethuntsa, and M.-S. Alouini, "Mixed $\mathrm{rf} /$ fso communications with outdated-csi-based relay selection under double generalized gamma turbulence, generalized pointing errors and nakagami-m fading," IEEE Transactions on Wireless Communications, 2019, doi: 10.1109/TWC.2019.2954866.

[19] E. Zedini, H. Soury, and M.-S. Alouini, "On the performance analysis of dual-hop mixed FSO/RF systems," IEEE Transactions on Wireless Communications, vol. 15, no. 5, pp. 3679-3689, 2016.

[20] S. Anees and M. R. Bhatnagar, "Performance of an amplify-andforward dual-hop asymmetric RF-FSO communication system," Journal of Optical Communications and Networking, vol. 7, no. 2, pp. 124-135, 2015.

[21] K. P. Peppas, A. N. Stassinakis, H. E. Nistazakis, and G. S. Tombras, "Capacity analysis of dual-hop amplify-and-forward relayed free-space optical communication systems over turbulence channel with pointing errors," IEEE/OSA Journal of Optical Communications and Networking, vol. 5, no. 9, pp. 1032-1042, 2013.

[22] E. Zedini, H. Soury, and M.-S. Alouini, "Dual-hop FSO transmission systems over Gamma-Gamma turbulence with pointing errors," IEEE Transactions on Wireless Communications, vol. 16, no. 2, pp. 784-796, 2016.

[23] A. M. Mathai and R. K. Saxena, The H function with applications in statistics and other disciplines. Wiley Eastern, New Dheli and Halsted Press, 1978, New York.

[24] A. M. Mathai, R. K. Saxena, and H. J. Haubold, The H-function: theory and applications. Springer Science \& Business Media, 2009.

[25] O. S. Badarneh, "Exact closed-form expression for end-to-end average SNR of dual-hop amplify-and-forward in wireless relaying systems," Wireless Personal Communications, vol. 86, no. 2, pp. 1023-1036, 2016.

[26] S. Anees and M. R. Bhatnagar, "Information theoretic analysis of a dualhop fixed gain AF based mixed RF-FSO system," in 2015 IEEE 26th Annual International Symposium on Personal, Indoor and Mobile Radio Communications (PIMRC). IEEE, 2015, pp. 927-931.

[27] _ , "Information theoretic analysis of a dual-hop DF based FSO communication system," in 2016 IEEE 83rd Vehicular Technology Conference (VTC Spring). IEEE, 2016, pp. 1-5.

[28] H. Chergui, M. Benjillali, and M.-S. Alouini, "Rician $K$-factor-based analysis of XLOS service probability in 5G outdoor ultra-dense networks," vol. 8, no. 2, pp. 428-431, 2018.

[29] H. R. Alhennawi, M. M. El Ayadi, M. H. Ismail, and H.-A. M. Mourad, "Closed-form exact and asymptotic expressions for the symbol error rate and capacity of the H-function fading channel," IEEE Transactions on Vehicular Technology, vol. 65, no. 4, pp. 1957-1974, 2016.

[30] I. S. Ansari, S. Al-Ahmadi, F. Yilmaz, M.-S. Alouini, and H. Yanikomeroglu, "A new formula for the BER of binary modulations with dual-branch selection over generalized-K composite fading channels," IEEE Transactions on Communications, vol. 59, no. 10, pp. 2654-2658, 2011.

[31] K. P. Peppas, "A new formula for the average bit error probability of dual-hop amplify-and-forward relaying systems over generalized shadowed fading channels," IEEE Wireless Communications Letters, vol. 1, no. 2, pp. 85-88, 2012.

[32] A. Soulimani, M. Benjillali, H. Chergui, and D. B. da Costa, "On multihop Weibull-fading communications: Performance analysis framework and applications," arXiv preprint arXiv:1610.08535, 2016.

[33] H. Chergui, M. Benjillali, and S. Saoudi, "Performance analysis of project-and-forward relaying in mixed MIMO-pinhole and Rayleigh dual-hop channel," IEEE Communications Letters, vol. 20, no. 3, pp. 610-613, 2016

[34] H. Lei, I. S. Ansari, G. Pan, B. Alomair, and M.-S. Alouini, "Secrecy capacity analysis over $\alpha-\mu$ fading channels," IEEE Communication Letters, vol. 21, no. 6, pp. 1445-1448, 2017.

[35] C. R. N. Da Silva, N. Simmons, E. J. Leonardo, S. L. Cotton, and M. D. Yacoub, "Ratio of two envelopes taken from $\alpha-\mu, \eta-\mu$, and $\kappa$ $\mu$ variates and some practical applications," IEEE Access, vol. 7, pp. 54 449-54 463, 2019.

[36] C. S. Meijer, "Multiplikationstheoreme für die funktion $G_{p, q}^{m, n}(z)$," in Proc. Ned. Akad. Wet., vol. 44, 1941, pp. 1062-1070.

[37] M. Hasna and M. Alouini, "End-to-end performance analysis of transmission systems with relays over Rayleigh-fading channels," IEEE Transactions on Wireless Communications, vol. 2, no. 6, pp. 1126-1131, 2003.

[38] C. S. Meijer, "Expansion theorems for the G-function. I," in Indagationes Mathematicae (Proceedings), vol. 55. Elsevier, 1952, pp. 369-379.

[39] N. Wang, "Parameter estimation for wireless fading and turbulence channels," Ph.D. dissertation, University of British Columbia, 2010.

[40] A. C. Cohen Jr, "Estimating parameters of logarithmic-normal distributioms by maximum likelihood," Journal of American Statistical Association, vol. 46, no. 254, pp. 206-212, 1951. 
[41] D. G. Voelz and X. Xiao, "Metric for optimizing spatially partially coherent beams for propagation through turbulence," Optical Engineering, vol. 48, no. 3, p. 036001, 2009.

[42] A. Prudnikov, Y. Brychkov, and O. Marichev, Integrals and Series, vol 3: more special functions. CRC Press, 1999, Boca Raton, FL USA.

[43] C. S. Meijer, "Expansion theorems for the G-function. II," in Indagationes Mathematicae (Proceedings), vol. 55. Elsevier, 1952, pp. 483487.

[44] S. B. Yakubovich and T. H. Nguyen, The Double Mellin-barnes Type Integrals And Their Application To Convolution Theory. World Scientific, 1992, vol. 6.

[45] Y. Aborahama, M. H. Ismail, and M. Hassan, "Novel methods for generating fox's H-function distributed random variables with applications," IEEE Transactions on Vehicular Technology, vol. 68, no. 7, pp. 71507154, 2019.

[46] C. E. Shannon, "A mathematical theory of communication," The Bell System Technical Journal, vol. 27, pp. 379-423, 1948.

[47] A. Annamalai, R. Palat, and J. Matyjas, "Estimating ergodic capacity of cooperative analog relaying under different adaptive source transmission techniques," in 2010 IEEE Sarnoff Symposium. IEEE, 2010, pp. 1-5.

[48] K. S. Yoo, C. S. Paschalis, L. C. Simon, M. Sami, F. J. Lopez-Martinez, J. M. Romero-Jerez, and G. K. Karagiannidis, "A comprehensive analysis of the achievable channel capacity in $\mathcal{F}$ composite fading channels," IEEE Access, vol. 7, pp. 34 078-34094, 2019.

[49] R. K. Mallik, M. Z. Win, J. W. Shao, M.-S. Alouini, and A. J. Goldsmith, "Channel capacity of adaptive transmission with maximal ratio combining in correlated rayleigh fading," IEEE Transactions on Wireless Communications, vol. 3, no. 4, pp. 1124-1133, 2004.

[50] W. Gappmair, "Further results on the capacity of free-space optical channels in turbulent atmosphere," IET Communications, vol. 5, no. 9, pp. 1262-1267, 2011.

[51] J. Zhang, M. Matthaiou, Z. Tan, and H. Wang, "Performance analysis of digital communication systems over composite $\eta-\mu$ /gamma fading channels," IEEE Transactions on Vehicular Technology, vol. 61, no. 7, pp. 3114-3124, 2012.

[52] A. Lapidoth, S. M. Moser, and M. A. Wigger, "On the capacity of free-space optical intensity channels," IEEE Transactions on Information Theory, vol. 55, no. 10, pp. 4449-4461, 2009.

[53] D. Wu and R. Negi, "Effective capacity : a wireless link model for support of quality of service," IEEE Transactions on Wireless Communications, vol. 2, no. 4, pp. 630-643, 2003.

[54] K. P. Peppas, P. T. Mathiopoulos, and J. Yanf, "On the effective capacity of amplify-and-forward multihop transmission over arbitrary and correlated fading channels," IEEE Wireless Communication Letters, vol. 5, no. 3, pp. 248-251, 2016.

[55] S. Efazati and P. Azmi, "Effective capacity maximization in multirelay transmission framework and power-allocation scheme," IEEE Transactions on Vehicular Technology, vol. 63, no. 4, pp. 1691-1702, 2013.

[56] G. Ozcan and M. C. Gursoy, "Effective capacity analysis of fixed-gain and variable-gain AF two-way relaying," in 2013 IEEE 78rd Vehicular Technology Conference (VTC Fall). IEEE, 2013, pp. 1-5.

[57] M. K. Simon and M.-S. Alouini, Digital communication over fading channels. JohnWiley \& Sons, 2005, vol. 95.

[58] F. Yilmaz and M.-S. Alouini, "A novel unified expression for the capacity and bit error probability of wireless communication systems over generalized fading channels," IEEE Transactions on Communications, vol. 60, no. 7, pp. 1862-1876, 2012.

[59] C. S. Meijer, "Expansion theorems for the G-function. IV," in Indagationes Mathematicae (Proceedings), vol. 56. Elsevier, 1953, pp. 187193.

[60] S. Chen, W. Wang, X. Zhang, and Z. Sun, "Performance analysis of OSTBC transmission in amplifyand-forward cooperative relay networks," IEEE Transactions on Vehicular Technology, vol. 59, no. 1, pp. 105-113, 2009.

[61] S. Chen, W. Wang, X. Zhang, X. Zhang, M. Peng, and Y. Li, "Ergodic and outage capacity analysis of amplify-and-forward MIMO relay with OSTBCs," in 2010 IEEE Wireless Communication and Networking Conference. IEEE, 2010, pp. 1-6.

[62] P. Anghel and M. Kaveh, "Exact symbol error probability of a cooperative network in a Rayleigh-fading environment," IEEE Transactions on Wireless Communications, vol. 3, no. 5, pp. 1416-1421, 2004.

[63] K. P. Peppas, G. C. Alexadropoulos, and P. T. Mathiopoulos, "Performance analysis of dual-hop AF relaying systems over mixed $\eta-\mu$ and $\kappa$ $\mu$ fading channels," IEEE Transactions on Vehicular Technology, vol. 62, no. 7, pp. 3149-3163, 2013.

[64] I. Gradshteyn and I. Ryzhik, Table of Integrals, Series, and Products. Elsevier Science, 2014.
[65] F. W. Olver, D. W. Lozier, R. F. Boisvert, and C. W. Clark, NIST handbook of mathematical functions. Cambridge University Press, 2010.

[66] H. A. Suraweera, R. Louie, Y. Li, G. K. Karagiannidis, and B. Vucetic, "Two hop amplify-and-forward transmission in mixed Rayleigh and rician fading channels," IEEE Communications letters, vol. 13, no. 4, pp. 227-229, 2009.

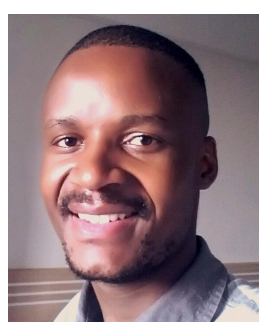

Tau Raphael Rasethuntsa (S'19) was born in Qoaling, Maseru, Lesotho. He matriculated from Azara Secondary School, Lenasia, Gauteng, Republic of South Africa at the top of his class in 2008. Mr Rasethuntsa received his BSc. First Class in Mathematics and Statistics from the National University of Lesotho, Roma, Maseru, Lesotho in 2014 and MSc. with distinction in Mathematical Engineering from Istanbul Technical University, Maslak, Istanbul, Turkey in 2017. He is currently with the department of Electrical and Computer Engineering at McMaster University, Hamilton, Ontario, Canada where he is pursuing his $\mathrm{PhD}$. His research interests include applied mathematics and statistics, particularly reliability as well as mathematical and statistical aspects of signal processing with special focus on the modeling and performance evaluation of wireless communication systems.

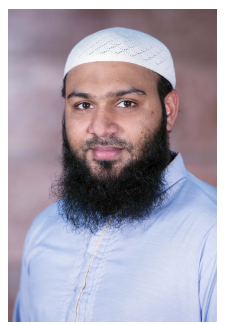

Imran Shafique Ansaria (S'07-M'15) received the B.Sc. degree in Computer Engineering from King Fahd University of Petroleum and Minerals (KFUPM) in 2009 (with First Honors) and M.Sc. and $\mathrm{PhD}$ degrees from King Abdullah University of Science and Technology (KAUST) in 2010 and 2015, respectively. Currently, since August 2018, he is a Lecturer (Assistant Professor) with University of Glasgow, Glasgow, UK. Prior to this, from November 2017 to July 2018, he was a Lecturer (Assistant Professor) with Global College of Engineering and Technology (GCET) (affiliated with University of the West of England (UWE), Bristol, UK). From April 2015 to November 2017, he was a Postdoctoral Research Associate (PRA) with Texas A\&M University at Qatar (TAMUQ). From May 2009 through Aug. 2009, he was a visiting scholar with Michigan State University (MSU), East Lansing, MI, USA, and from Jun. 2010 through Aug. 2010, he was a research intern with Carleton University, Ottawa, ON, Canada.

He has been affiliated with IEEE since 2007 and has served in various capacities. He is serving on the IEEE Nominations and Appointments (N\&A) Committee since 2020-2021 and IEEE Communication Society Young Professionals (ComSoc YP) Board since April 2016. He is part of the IEEE 5G Tech Focus Publications Editorial Board since Feb. 2017. He is an active reviewer for various IEEE Transactions and various other journals. He has served as a TPC for various IEEE conferences. He is a recipient of appreciation for an exemplary reviewer for IEEE Transaction on Communications (TCOM) in 2018 and 2016, a recipient of appreciation for an exemplary reviewer for IEEE Wireless Communications Letters (WCL) in 2017 and 2014, a recipient of TAMUQ ECEN Research Excellence Award 2016, 2017, a recipient of postdoctoral research award (PDRA) (first cycle) with Qatar national research foundation (QNRF) in 2014, a recipient of KAUST academic excellence award (AEA) in 2014, and a recipient of IEEE Richard E. Merwin student scholarship award in Jul. 2013.

Dr. Ansari has authored/co-authored 100+ journal and conference publications. He has co-organized the GRASNET'2016, 2017, 2018 workshops in conjunction with IEEE WCNC'2016, 2017 and IEEE Globecom 2018. His current research interests include free-space optics (FSO), underwater communications, physical layer secrecy issues, full duplex systems, reconfigurable intelligent surfaces (RIS) / intelligent reflective surfaces (IRS), and secure D2D applications for $5 \mathrm{G}+$ systems, among others. 